\title{
THE RATIONALITY OF THE MODULI SPACE OF CURVES OF GENUS 3 AFTER P. KATSYLO
}

\author{
CHRISTIAN BÖHNING
}

\begin{abstract}
This article is a survey of P. Katsylo's proof that the moduli space $\mathfrak{M}_{3}$ of smooth projective complex curves of genus 3 is rational. We hope to make the argument more comprehensible and transparent by emphasizing the underlying geometry in the proof and its key structural features.
\end{abstract}

\section{Contents}

1. Introduction

2. A remarkable $\left(\mathrm{SL}_{3} \mathbb{C}, \mathrm{SO}_{3} \mathbb{C}\right)$-section

2.1. $(G, H)$-sections and covariants

2.2. Binary quartics

2.3. From quintic to cubic equations

2.4. From cubic to quadratic equations

3. Further sections and inner projections

3.1. Binary quartics again and a $\left(\mathrm{PSL}_{2} \mathbb{C}, \mathfrak{S}_{4}\right)$-section $\quad 18$

3.2. Dividing by the action of $H \quad 23$

3.3. Inner projections and the "no-name" method 26

Appendix A. Collection of formulas for section $2 \quad 33$

Appendix B. Collection of formulas for section $3 \quad 37$

References $\quad 38$

\section{INTRODUCTION}

The question whether $\mathfrak{M}_{3}$ is a rational variety or not had been open for a long time until an affirmative answer was finally given by P. Katsylo in 1996. There is a well known transition in the behaviour of the moduli spaces $\mathfrak{M}_{g}$ of smooth projective complex curves of genus $g$ from unirational for small $g$ to general type for larger values of $g$; the moral reason that $\mathfrak{M}_{3}$ should have a good chance to be rational is that it is birational to a quotient of a projective space by a connected linear algebraic group. No variety of this form has been proved irrational 
up to now. More precisely, $\mathfrak{M}_{3}$ is birational to the moduli space of plane quartic curves for $\mathrm{PGL}_{3} \mathbb{C}$-equivalence. All the moduli spaces $C(d)$ of plane curves of given degree $d$ are conjectured to be rational (see [Dol2], p.162; in fact, there it is conjectured that all the moduli spaces of hypersurfaces of given degree $d$ in $\mathbb{P}^{n}$ for the $\mathrm{PGL}_{n+1} \mathbb{C}$-action are rational. I do not know if this conjecture should be attributed to Dolgachev or someone else).

There are heuristic reasons that the spaces $C(d)$ should be rational at least for all large enough values for $d$. Maybe it is not completely out of reach to prove this rigorously. We hope to return to this problem in the future. In any case one might hazard the guess that irregular behaviour of $C(d)$ is most likely to be found for small values of $d$, and showing rationality for $C(4)$ turned out to be exceptionally hard.

Katsylo's proof is long and computational, and, due to the importance of the result, it seems desirable to give a more accessible and geometric treatment of the argument.

This paper is divided into two main sections (sections 2 and 3 ) which are further divided into subsections. Section 2 treats roughly the contents of Katsylo's first paper [Kat1] and section 3 deals with his second paper Kat2].

Finally I would like to thank Professor Yuri Tschinkel for proposing the project and many useful discussions. Moreover, I am especially grateful to Professor Fedor Bogomolov with whom I discussed parts of the project and who provided a wealth of helpful ideas.

\section{A Remarkable $\left(\mathrm{SL}_{3} \mathbb{C}, \mathrm{SO}_{3} \mathbb{C}\right)$-SECtion}

2.1. $(G, H)$-sections and covariants. A general, i.e. nonhyperelliptic, smooth projective curve $C$ of genus 3 is realized as a smooth plane quartic curve via the canonical embedding, whence $\mathfrak{M}_{3}$ is birational to the orbit space $C(4):=\mathbb{P}\left(H^{0}\left(\mathbb{P}^{2}, \mathcal{O}(4)\right) / \mathrm{SL}_{3} \mathbb{C}\right.$. We remark that whenever one has an affine algebraic group $G$ acting on an irreducible variety $X$, then, according to a result of Rosenlicht, there exists a nonempty invariant open subset $X_{0} \subset X$ such that there is a geometric quotient for the action of $G$ on $X_{0}$ (cf. [Po-Vi], thm. 4.4). In the following we denote by $X / G$ any birational model of this quotient, i.e. any model of the field $\mathbb{C}(X)^{G}$ of invariant rational functions.

The number of methods to prove rationality of quotients of projective spaces by connected reductive groups is quite limited (cf. [Dol1] for an excellent survey). The only approach which our problem is immediately amenable to seems to be the method of $(G, H)$-sections. (There are two other points of view I know of: The first is based on the remark 
that if we have a nonsingular plane quartic curve $C$, the double cover of $\mathbb{P}^{2}$ branched along $C$ is a Del Pezzo surface of degree 2, and conversely, given a Del Pezzo surface $S$ of degree 2 , then $\left|-K_{S}\right|$ is a regular map which exhibits $S$ as a double cover of $\mathbb{P}^{2}$ branched along a plane quartic $C$; this sets up a birational isomorphism between $\mathfrak{M}_{3}$ and $\mathfrak{D P}(2)$, the moduli space of Del Pezzo surfaces of degree 2. We can obtain such an $S$ by blowing up 7 points in $\mathbb{P}^{2}$, and one can prove that $\mathfrak{D P}(2)$ is birational to the quotient of an open subset of $P_{2}^{7}:=\left(\mathbb{P}^{2}\right)^{7} / \mathrm{PGL}_{3} \mathbb{C}$, the configuration space of 7 points in $\mathbb{P}^{2}$ (which is visibly rational), modulo an action of the Weyl group $W\left(E_{7}\right)$ of the root system of type $E_{7}$ by Cremona transformations (note that $W\left(E_{7}\right)$ coincides with the permutation group of the $(-1)$-curves on $S$ that preserves the incidence relations between them). This group is a rather large finite group, in fact, it has order $2^{10} \cdot 3^{4} \cdot 5 \cdot 7$. This approach does not seem to have led to anything definite in the direction of proving rationality of $\mathfrak{M}_{3}$ by now, but see [D-O] for more information.

The second alternative, pointed out by I. Dolgachev, is to remark that $\mathfrak{M}_{3}$ is birational to $\mathfrak{M}_{3}^{\mathrm{ev}}$, the moduli space of genus 3 curves together with an even theta-characteristic; this is the content of the classical theorem due to G. Scorza. The latter space is birational to the space of nets of quadrics in $\mathbb{P}^{3}$ modulo the action of $\mathrm{SL}_{4} \mathbb{C}$, i.e. $\operatorname{Grass}\left(3, \operatorname{Sym}^{2}\left(\mathbb{C}^{4}\right)^{\vee}\right) / \mathrm{SL}_{4} \mathbb{C}$. See [Dol3], 6.4.2, for more on this. Compare also [Kat0, where the rationality of the related space $\operatorname{Grass}\left(3, \operatorname{Sym}^{2}\left(\mathbb{C}^{5}\right)^{\vee}\right) / \mathrm{SL}_{5} \mathbb{C}$ is proven; this proof, however, cannot be readily adapted to our situation, the difficulty seems to come down to that 4 , in contrast to 5 , is even).

Definition 2.1.1. Let $X$ be an irreducible variety with an action of a linear algebraic group $G, H<G$ a subgroup. An irreducible subvariety $Y \subset X$ is called a $(G, H)$-section of the action of $G$ on $\mathrm{X}$ if

(1) $\overline{G \cdot Y}=X$

(2) $H \cdot Y \subset Y$;

(3) $g \in G, g Y \cap Y \neq \emptyset \Longrightarrow g \in H$.

In this situation $H$ is the normalizer $N_{G}(Y):=\{g \in G \mid g Y \subset Y\}$ of $Y$ in $G$. The following proposition collects some properties of $(G, H)$ sections.

Proposition 2.1.2. (1) The field $\mathbb{C}(X)^{G}$ is isomorphic to the field $\mathbb{C}(Y)^{H}$ via restriction of functions to $Y$. 
(2) Let $Z$ and $X$ be $G$-varieties, $f: Z \rightarrow X$ a dominant $G$ morphism, $Y$ a $(G, H)$-section of $X$, and $Y^{\prime}$ an irreducible component of $f^{-1}(Y)$ that is $H$-invariant and dominates $Y$. Then $Y^{\prime}$ is a $(G, H)$-section of $Z$.

Part (2) of the proposition suggests that, to simplify our problem of proving rationality of $C(4)$, we should look at covariants $\operatorname{Sym}^{4}\left(\mathbb{C}^{3}\right)^{\vee} \rightarrow$ $\operatorname{Sym}^{2}\left(\mathbb{C}^{3}\right)^{\vee}$ of low degree $\left(\mathbb{C}^{3}\right.$ is the standard representation of $\left.\mathrm{SL}_{3} \mathbb{C}\right)$. The highest weight theory of Cartan-Killing allows us to decompose $\operatorname{Sym}^{i}\left(\operatorname{Sym}^{4}\left(\mathbb{C}^{3}\right)^{\vee}\right), i \in \mathbb{N}$, into irreducible subrepresentations (this is best done by a computer algebra system, e.g. Magma) and pick the smallest $i$ such that $\operatorname{Sym}^{2}\left(\mathbb{C}^{3}\right)^{\vee}$ occurs as an irreducible summand. This turns out to be 5 and $\operatorname{Sym}^{2}\left(\mathbb{C}^{3}\right)^{\vee}$ occurs with multiplicity 2 .

For nonnegative integers $a, b$ we denote by $V(a, b)$ the irreducible $\mathrm{SL}_{3} \mathbb{C}$ module whose highest weight has numerical labels $a, b$.

Let us now describe the two resulting independent covariants

$$
\alpha_{1}, \alpha_{2}: V(0,4) \rightarrow V(0,2)
$$

of order 2 and degree 5 geometrically. We follow a classical geometric method of Clebsch to pass from invariants of binary forms to contravariants of ternary forms (see G-Y], §215). The covariants $\alpha_{1}, \alpha_{2}$ are described in Salmon's treatise [Sal], p. 261, and p. 259, cf. also Dix, p. 280-282. We start by recalling the structure of the ring of $\mathrm{SL}_{2} \mathbb{C}$-invariants of binary quartics ([Muk], section 1.3, [Po-Vi], section $0.12)$.

\subsection{Binary quartics. Let}

$$
f_{4}=\xi_{0} x_{0}^{4}+4 \xi_{1} x_{0}^{3} x_{1}+6 \xi_{2} x_{0}^{2} x_{1}^{2}+4 \xi_{3} x_{0} x_{1}^{3}+\xi_{4} x_{1}^{4}
$$

be a general binary quartic form. The invariant algebra $R=\mathbb{C}\left[\xi_{0}, \ldots, \xi_{4}\right]^{\mathrm{SL}_{2} \mathbb{C}}$ is freely generated by two homogeneous invariants $g_{2}$ and $g_{3}$ (where subscripts indicate degrees):

$$
\begin{gathered}
g_{2}(\xi)=\operatorname{det}\left(\begin{array}{ll}
\xi_{0} & \xi_{2} \\
\xi_{2} & \xi_{4}
\end{array}\right)-4 \operatorname{det}\left(\begin{array}{ll}
\xi_{1} & \xi_{2} \\
\xi_{2} & \xi_{3}
\end{array}\right), \\
g_{3}(\xi)=\operatorname{det}\left(\begin{array}{lll}
\xi_{0} & \xi_{1} & \xi_{2} \\
\xi_{1} & \xi_{2} & \xi_{3} \\
\xi_{2} & \xi_{3} & \xi_{4}
\end{array}\right) .
\end{gathered}
$$

If we identify $f_{4}$ with its zeroes $z_{1}, \ldots, z_{4} \in \mathbb{P}^{1}=\mathbb{C} \cup\{\infty\}$ and write

$$
\lambda=\frac{\left(z_{1}-z_{3}\right)\left(z_{2}-z_{4}\right)}{\left(z_{1}-z_{4}\right)\left(z_{2}-z_{3}\right)}
$$


for the cross-ratio, then

$$
\begin{gathered}
g_{3}=0 \Longleftrightarrow \lambda=-1,2, \text { or } \frac{1}{2}, \\
g_{2}=0 \Longleftrightarrow \lambda=-\omega \text { or }-\omega^{2} \text { with } \omega=e^{\frac{2 \pi i}{3}},
\end{gathered}
$$

the first case being commonly referred to as harmonic cross-ratio, the second as equi-anharmonic cross-ratio (see [Cl], p. 171; the terminology varies a lot among different authors, however).

Clebsch's construction is as follows: Let $x, y, z$ be coordinates in $\mathbb{P}^{2}$, and let $u, v, w$ be coordinates in the dual projective plane $\left(\mathbb{P}^{2}\right)^{\vee}$. Let $\varphi=a x^{4}+4 b x^{3} y^{4}+\ldots$ be a general ternary quartic. We want to consider those lines in $\mathbb{P}^{2}$ such that their intersection with the associated quartic curve $C_{\varphi}$ is a set of points whose cross-ratio is harmonic resp. equianharmonic. Writing a line as $u x+v y+w z=0$ and substituting in (2) resp. (3), we see that in the equi-anharmonic case we get a quartic in $\left(\mathbb{P}^{2}\right)^{\vee}$, and in the harmonic case a sextic. More precisely this gives us two $\mathrm{SL}_{3} \mathbb{C}$-equivariant polynomial maps

$$
\begin{aligned}
& \sigma: V(0,4) \rightarrow V(0,4)^{\vee}, \\
& \psi: V(0,4) \rightarrow V(0,6)^{\vee},
\end{aligned}
$$

and $\sigma$ is homogeneous of degree 2 in the coefficients of $\varphi$ whereas $\psi$ is homogeneous of degree 3 in the coefficients of $\varphi$ (we say $\sigma$ is a contravariant of degree 2 on $V(0,4)$ with values in $V(0,4)$, and analogously for $\psi$ ). Finally we have the Hessian covariant of $\varphi$ :

$$
\text { Hess : } V(0,4) \rightarrow V(0,6)
$$

which associates to $\varphi$ the determinant of the matrix of second partial derivatives of $\varphi$. It is of degree 3 in the coefficients of $\varphi$.

We will now cook up $\alpha_{1}, \alpha_{2}$ from $\varphi, \sigma, \psi$, Hess: Let $\varphi$ operate on $\psi$; by this we mean that if $\varphi=a x^{4}+4 b x^{3} y+\ldots$ then we act on $\psi$ by the differential operator

$$
a \frac{\partial^{4}}{\partial u^{4}}+4 b \frac{\partial^{4}}{\partial u^{3} \partial v}+\ldots
$$

(i.e. we replace a coordinate by partial differentiation with respect to the dual coordinate). In this way we get a contravariant $\rho$ of degree 4 on $V(0,4)$ with values in $V(0,2)$. If we operate with $\rho$ on $\varphi$ we get $\alpha_{1}$. We obtain $\alpha_{2}$ if we operate with $\sigma$ on Hess.

This is a geometric way to describe $\alpha_{1}, \alpha_{2}$. For every $c=\left[c_{1}: c_{2}\right] \in \mathbb{P}^{1}$ we get in this way a rational map

$$
f_{c}=c_{1} \alpha_{1}+c_{2} \alpha_{2}: \mathbb{P}(V(0,4)) \rightarrow \mathbb{P}(V(0,2)) .
$$


For the special quartics

$$
\varphi=a x^{4}+b y^{4}+c z^{4}+6 f y^{2} z^{2}+6 g z^{2} x^{2}+6 h x^{2} y^{2}
$$

the quantities $\alpha_{1}$ and $\alpha_{2}$ were calculated by Salmon in [Sal], p. $257 \mathrm{ff}$. We reproduce the results here for the reader's convenience. Put

$$
\begin{aligned}
L:=a b c, P & :=a f^{2}+b g^{2}+c h^{2}, \\
R & :=f g h ;
\end{aligned}
$$

Then

$$
\begin{gathered}
\alpha_{1}=(3 L+9 P+10 R)\left(a f x^{2}+b g y^{2}+c h z^{2}\right)+ \\
(10 L+2 P+4 R)\left(g h x^{2}+h f y^{2}+f g z^{2}\right) \\
-12\left(a^{2} f^{3} x^{2}+b^{2} g^{3} y^{2}+c^{2} h^{3} z^{2}\right) \\
\alpha_{2}=(L+3 P+30 R)\left(a f x^{2}+b g y^{2}+c h z^{2}\right)+ \\
(10 L-6 P-12 R)\left(g h x^{2}+h f y^{2}+f g z^{2}\right) \\
-4\left(a^{2} f^{3} x^{2}+b^{2} g^{3} y^{2}+c^{2} h^{3} z^{2}\right) .
\end{gathered}
$$

Note that the covariant conic $-\frac{1}{20}\left(\alpha_{1}-3 \alpha_{2}\right)$ looks a little simpler.

Let us see explicitly, using (8)-(11), that $f_{c}$ is dominant for every $c \in \mathbb{P}^{1}$; for $a=b=c=f=g=h=1$ we get $\alpha_{1}=48\left(x^{2}+y^{2}+z^{2}\right)$, $\alpha_{2}=16\left(x^{2}+y^{2}+z^{2}\right)$, so the image of $\varphi$ under $f_{c}$ in this case is a nonsingular conic unless $c=[-1: 3]$. But for $a=1, b=c=0, f=g=h=1$ we obtain $\alpha_{1}=13 x^{2}+6 y^{2}+6 z^{2}, \alpha_{2}=11 x^{2}-18 y^{2}-18 z^{2}$, and for these values $-\alpha_{1}+3 \alpha_{2}$ defines a nonsingular conic.

Let $\mathcal{L}_{c}$ be the linear system generated by 6 quintics which defines $f_{c}$ and let $B_{c}$ be its base locus; thus $U_{c}:=\mathbb{P}(V(0,4)) \backslash B$ is an $\mathrm{SL}_{3} \mathbb{C}$-invariant open set, and if $f_{c, 0}:=\left.f_{c}\right|_{U_{c}}$, then $X_{c}:=f_{c, 0}^{-1}\left(\mathbb{C} h_{0}\right)$, where $h_{0}$ defines a non-singular conic, is a good candidate for an $\left(\mathrm{SL}_{3} \mathbb{C}, \mathrm{SO}_{3} \mathbb{C}\right)$-section of $U_{c}$. We choose $h_{0}=x z-y^{2}$.

Proposition 2.2.1. $X_{c}$ is a smooth irreducible $\mathrm{SO}_{3} \mathbb{C}$-invariant variety, $\overline{\mathrm{SL}_{3} \mathbb{C} \cdot X}=\mathbb{P}(V(0,4))$, and the normalizer of $X_{c}$ in $\mathrm{SL}_{3} \mathbb{C}$ is exactly $\mathrm{SO}_{3} \mathbb{C} . X_{c}$ is an $\left(\mathrm{SL}_{3} \mathbb{C}, \mathrm{SO}_{3} \mathbb{C}\right)$-section of $U_{c}$.

Proof. The $\mathrm{SO}_{3} \mathbb{C}$-invariance of $X_{c}$ follows from its construction. We show that the differential $d\left(f_{c, 0}\right)_{x}$ is surjective for all $x \in X_{c}$ : In fact,

$$
d\left(f_{c, 0}\right)_{x}\left(T_{x} U_{c}\right) \supset d\left(f_{c, 0}\right)_{x}\left(\mathfrak{s l}_{3}(x)\right)=\mathfrak{s l}_{3}\left(f_{c, 0}(x)\right)=T_{\mathbb{C} h_{0}} \mathbb{P} V(0,2)
$$

Here $\mathfrak{s l}_{3}(x)$ denotes the tangent space to the $\mathrm{SL}_{3} \mathbb{C}$-orbit of $x$ in $U_{c}$, i.e. if $O_{x}: \mathrm{SL}_{3} \mathbb{C} \rightarrow U_{c}$ is the map with $O_{x}(g)=g x$, then we get a map $d\left(O_{x}\right)_{e}: \mathfrak{s l}_{3} \rightarrow T_{x} U_{c}$, and $\mathfrak{s l}_{3}(x):=\left\{d\left(O_{x}\right)_{e}(\xi) \mid \xi \in \mathfrak{s l}_{3}\right\}$. Hence $X_{c}$ is 
smooth.

Assume $X_{c}$ were reducible, let $X_{1}$ and $X_{2}$ be two irreducible components. By prop. 2.1.2 (2) and the irreducibility of the group $\mathrm{SO}_{3} \mathbb{C}$, $X_{1}$ and $X_{2}$ are $\left(\mathrm{SL}_{3} \mathbb{C}, \mathrm{SO}_{3} \mathbb{C}\right)$-sections of $U_{c}$, so we can find $g \in \mathrm{SL}_{3} \mathbb{C}$, $x_{1} \in X_{1}, x_{2} \in X_{2}$, such that $g x_{1}=x_{2}$. But then, by the $\mathrm{SL}_{3} \mathbb{C}$ equivariance of $f_{c, 0}, g$ stabilizes $\mathbb{C} h_{0}$ and is thus in $\mathrm{SO}_{3} \mathbb{C}$. But, again by the irreducibility of $\mathrm{SO}_{3} \mathbb{C}, x_{2}$ is also a point of $X_{1}$, i.e. $X_{1}$ and $X_{2}$ meet. This contradicts the smoothness of $X_{c}$.

The trouble is that, if $\bar{X}_{c}$ is the closure of $X_{c}$ in $\mathbb{P}(V(0,4))$, then $\bar{X}_{c}$ is an irreducible component of the intersection of 5 quintics. To eventually prove rationality, however, we would like to have some equations of lower degree. This can be done for special $c$.

2.3. From quintic to cubic equations. If $\Gamma_{f_{c}} \subset \mathbb{P} V(0,4) \times \mathbb{P} V(0,2)$ is the graph of $f_{c}$, it is natural to look for $\mathrm{SL}_{3} \mathbb{C}$-equivariant maps

$$
\vartheta: V(0,4) \times V(0,2) \rightarrow V^{\prime}
$$

where $V^{\prime}$ is another $\mathrm{SL}_{3} \mathbb{C}$-representation, $\vartheta$ is a homogeneous polynomial map in both factors $V(0,4), V(0,2)$, of low degree, say $d$, in the first factor, linear in the second, and such that $\Gamma_{f_{c}}$ is an irreducible component of $\{(x, y) \in \mathbb{P} V(0,4) \times \mathbb{P} V(0,2) \mid \vartheta(x, y)=0\}$. If $V^{\prime}$ is irreducible, there is an easy way to tell if $\vartheta$ vanishes on $\Gamma_{f_{c}}$ for some $c \in \mathbb{P}^{1}$ : This will be the case if $V^{\prime}$ occurs with multiplicity one in $\operatorname{Sym}^{d+5} V(0,4)$. Here is the result.

Definition 2.3.1. Let $\Psi: V(0,4) \rightarrow V(2,2)$ be the up to factor unique $\mathrm{SL}_{3} \mathbb{C}$-equivariant, homogeneous of degree 3 polynomial map with the indicated source and target spaces, and let $\Phi: V(2,2) \times V(0,2) \rightarrow$ $V(2,1)$ be the up to factor unique bilinear $\mathrm{SL}_{3} \mathbb{C}$-equivariant map. Define $\Theta: V(0,4) \times V(0,2) \rightarrow V(2,1)$ by $\Theta(x, y):=\Phi(\Psi(x), y)$.

Remark 2.3.2. The existence and essential uniqueness of the maps of definition 2.3.1 can be easily deduced from known (and implemented in Magma) decomposition laws for $\mathrm{SL}_{3} \mathbb{C}$-representations. That they are only determined up to a nonzero constant factor will never bother us, and we admit this ambiguity in notation. The explicit form of $\Psi, \Phi$, $\Theta$ will be needed later for checking certain non-degeneracy conditions through explicit computation. They can be found in Appendix A, formulas (64), (65).

Theorem 2.3.3. (1) The linear map $\Theta(f, \cdot): V(0,2) \rightarrow V(2,1)$ has one-dimensional kernel for $f$ in an open dense subset $V_{0}$ of $V(0,4)$, and, in particular, $\operatorname{ker} \Theta\left(h_{0}^{2}, \cdot\right)=\mathbb{C} h_{0}$. 
(2) For some $c_{0} \in \mathbb{P}^{1}, \Gamma_{f_{c_{0}}}$ is an irreducible component of $\{\Theta(x, y)=$ $0\} \subset \mathbb{P} V(0,4) \times \mathbb{P} V(0,2)$.

(3) $\overline{X_{c_{0}}} \subset \mathbb{P} V(0,4)$ coincides with the closure $\bar{X}$ in $\mathbb{P} V(0,4)$ of the preimage $X$ of $h_{0}$ under the morphism from $\mathbb{P} V_{0} \rightarrow \mathbb{P} V(0,2)$ given by $f \mapsto \operatorname{ker} \Theta(f, \cdot)$, and is thus an irreducible component of the algebraic set $\left\{\mathbb{C} f \mid \Phi\left(\Psi(f), h_{0}\right)=0\right\} \subset \mathbb{P} V(0,4)$ defined by 15 cubic equations.

(4) The rational map $\Psi: \mathbb{P} V(0,4) \rightarrow \overline{\Psi \mathbb{P} V(0,4)} \subset \mathbb{P} V(2,2)$ as well as its restriction to $X$ are birational isomorphisms unto their images.

Proof. (1): One checks that $V(2,1)$ occurs with multiplicity one in the decomposition of $\operatorname{Sym}^{8} V(0,4)$. Thus for some $c_{0} \in \mathbb{P}^{1}$, we have $\Theta\left(f,\left(c_{0,1} \alpha_{1}+c_{0,2} \alpha_{2}\right)(f)\right)=0$ for all $f \in V(0,4)$. The fact that ker $\Theta\left(h_{0}^{2}, \cdot\right)=\mathbb{C} h_{0}$ follows from a direct computation using the explicit form of $\Theta$. Thus, by upper-semicontinuity, (1) follows.

(2): We have seen in (1) that $\Gamma_{f_{c_{0}}}$ is contained in $\{\Theta(x, y)=0\}$. Again by $(1)$,

$$
\begin{gathered}
\Gamma_{f_{c_{0}}} \cap\left(\left(U_{c_{0}} \cap \mathbb{P} V_{0}\right) \times \mathbb{P} V(0,2)\right)= \\
\{\Theta(x, y)=0\} \cap\left(\left(U_{c_{0}} \cap \mathbb{P} V_{0}\right) \times \mathbb{P} V(0,2)\right),
\end{gathered}
$$

and (2) follows.

(3) follows from to (2) and the definition of $X_{c_{0}}$.

(4): Since $X$ is an $\left(\mathrm{SL}_{3} \mathbb{C}, \mathrm{SO}_{3} \mathbb{C}\right)$-section of $\mathbb{P} V_{0}$, it suffices to prove that the $\mathrm{SL}_{3} \mathbb{C}$-equivariant rational map $\Psi: \mathbb{P} V(0,4) \rightarrow \overline{\Psi \mathbb{P} V(0,4)}$ (defined e.g. in the point $\mathbb{C} h_{0}^{2}$ ) is birational. We will do this by writing down an explicit rational inverse. To do this, remark that $V(a, b)$ sits as an $\mathrm{SL}_{3} \mathbb{C}$-invariant linear subspace inside $\operatorname{Sym}^{a} \mathbb{C}^{3} \otimes \operatorname{Sym}^{b}\left(\mathbb{C}^{3}\right)^{\vee}$ (it has multiplicity one in the decomposition into irreducibles), thus elements of $V(a, b)$ may be viewed as tensors $x=\left(x_{j_{1}, \ldots, j_{a}}^{i_{1}, i_{b}}\right) \in T_{a}^{b} \mathbb{C}^{3}$, covariant of order $b$ and contravariant of order $a$, or of type $\left(\begin{array}{l}b \\ a\end{array}\right)$. The inverse of the determinant tensor $\operatorname{det}^{-1}$ is thus in $T_{3}^{0} \mathbb{C}^{3}$. For $f \in$ $V(0,4)$ and $g \in V(2,2)$ one defines a bilinear $\mathrm{SL}_{3} \mathbb{C}$-equivariant map $\alpha: V(0,4) \times V(2,2) \rightarrow \operatorname{Sym}^{2} \mathbb{C}^{3} \otimes \operatorname{Sym}^{3}\left(\mathbb{C}^{3}\right)^{\vee},(f, g) \mapsto \alpha(f, g)$, as the contraction

$$
s_{j_{1} j_{2}}^{i_{1} i_{2} i_{3}}:=f^{i_{1} i_{2} i_{4} i_{5}} g_{i_{5} j_{1}}^{i_{6} i_{3}} \operatorname{det}_{j_{2} i_{4} i_{6}}^{-1},
$$

followed by the symmetrization map. One checks that $\mathrm{Sym}^{2} \mathbb{C}^{3} \otimes$ $\operatorname{Sym}^{3}\left(\mathbb{C}^{3}\right)^{\vee}$ decomposes as $V(2,3) \oplus V(1,2) \oplus V(0,1)$, but $\operatorname{Sym}^{4} V(0,4)$ does not contain these as subrepresentations (use Magma), so $\alpha(f, \Psi(f))=$ 0 for all $f \in V(0,4)$. But the explicit forms of $\Psi$ and $\alpha$ show that ker $\alpha\left(\cdot, \Psi\left(h_{0}^{2}\right)\right)=\mathbb{C} h_{0}^{2}$, whence, by upper-semicontinuity, the dimension 
of the kernel of $\alpha(\cdot, \Psi(f))$ is one for all $f$ in a dense open subset of $V(0,4)$, and the rational map $\Psi: \mathbb{P} V(0,4) \rightarrow \overline{\Psi \mathbb{P} V(0,4)} \subset \mathbb{P} V(2,2)$ has the rational inverse $\Psi(f) \mapsto \operatorname{ker} \alpha(\cdot, \Psi(f))$.

Remark 2.3.4. It would probably be illuminating to have a geometric interpretation of the covariant $\Psi: V(0,4) \rightarrow V(2,2)$ given above similar to the one for $\alpha_{1}, \alpha_{2}$ in subsection 2.2. Though there is a huge amount of classical projective geometry attached to plane quartics, I have been unable to find such a geometric description.

Clearly, $\Psi$ vanishes on the cone of dominant vectors in $V(0,4)$, and one may check, using the explicit formula for $\Psi$ in Appendix A (64), that $\Psi$ also vanishes on the $\mathrm{SL}_{3} \mathbb{C}$-orbit of the degree 4 forms in two variables, $x$ and $y$, say. However, this, together with the fact that $\Psi$ is of degree 3 , is not enough to characterize $\Psi$ since the same holds also for e.g. the Hessian covariant.

2.4. From cubic to quadratic equations. We have to fix some further notation.

Definition 2.4.1. (1) $Z$ is the affine cone in $V(2,2)$ over $\overline{\Psi(X)} \subset$ $\mathbb{P} V(2,2)$.

(2) $L$ is the linear subspace $L:=\left\{g \in V(2,2) \mid \Phi\left(g, h_{0}\right)=0\right\} \subset$ $V(2,2)$.

(3) $\epsilon: V(0,4) \times V(0,2) \rightarrow V(2,2)$ is the unique (up to a nonzero factor) nontrivial $\mathrm{SL}_{3} \mathbb{C}$-equivariant bilinear map with the indicated source and target spaces (the explicit form is in Appendix A (66)).

(4) $\zeta: V(0,4) \times V(0,2) \rightarrow V(1,1)$ is the unique (up to factor) nontrivial $\mathrm{SL}_{3} \mathbb{C}$-equivariant map with the property that it is homogeneous of degree 2 in both factors of its domain (cf. Appendix A (67) for the explicit description). We put $\Gamma:=\zeta\left(\cdot, h_{0}\right)$ : $V(0,4) \rightarrow V(1,1)$.

Let us state explicitly what we are heading towards:

The affine cone $Z$ over the birational modification $\overline{\Psi(X)}$ of our $\left(\mathrm{SL}_{3} \mathbb{C}, \mathrm{SO}_{3} \mathbb{C}\right.$ )-section $X \subset \mathbb{P} V_{0} \subset \mathbb{P V}(0,4)$ (whose closure in $\mathbb{P} V(0,4)$ was seen to be an irreducible component of an algebraic set defined by 15 cubic equations) has the following wonderful properties: $Z$ lies in $L$, the linear map $\epsilon\left(\cdot, h_{0}\right): V(0,4) \rightarrow V(2,2)$ restricts to an $\mathrm{SO}_{3} \mathbb{C}$-equivariant isomorphism between $V(0,4)$ and $L$, and if, via this isomorphism, we transport $Z$ into $V(0,4)$ and call this $Y$, then the equations for $Y$ are given by 
$\Gamma$ ! More precisely, $Y$ is the unique irreducible component of $\Gamma^{-1}(0)$ passing through the point $h_{0}^{2}$, and $\Gamma$ maps $V(0,4)$ into a five-dimensional $\mathrm{SO}_{3} \mathbb{C}$-invariant subspace of $V(1,1)$ !

Thus, if we have carried out this program, $Y$ (or $Z$ ) will be proven to be an irreducible component of an algebraic set defined by 5 quadratic equations! This seems quite miraculous, but a satisfactory explanation why this happens probably requires an answer to the problem raised in remark 2.3.4.

We start with some preliminary observations: It is clear that $Z \subset L$ and $\mathbb{C}(\mathbb{P} V(0,4))^{\mathrm{SL}_{3} \mathbb{C}} \simeq \mathbb{C}(Z)^{\mathrm{SO}_{3} \mathbb{C} \times \mathbb{C}^{*}}, \mathbb{C}^{*}$ acting by homotheties. In the following, we need the decomposition into irreducibles of $\mathrm{SL}_{3} \mathbb{C}$-modules such as $V(2,2), V(2,1), V(1,1)$ and $V(0,4)$ as $\mathrm{SO}_{3} \mathbb{C}$-modules. The patterns according to which irreducible representations of a complex semi-simple algebraic group decompose when restricted to a smaller semi-simple subgroup are generally known as branching rules. In our case the answer is

$$
\begin{gathered}
V(2,2)=V(2,2)_{8} \oplus V(2,2)_{6} \oplus V(2,2)_{4} \oplus V(2,2)_{4}^{\prime} \oplus V(2,2)_{0}, \\
V(2,1)=V(2,1)_{6} \oplus V(2,1)_{4} \oplus V(2,1)_{2}, \\
V(1,1)=V(1,1)_{4} \oplus V(1,1)_{2}, \\
V(0,4)=V(0,4)_{8} \oplus V(0,4)_{4} \oplus V(0,4)_{0} .
\end{gathered}
$$

Here the subscripts indicate the numerical label of the highest weight of the respective $\mathrm{SO}_{3} \mathbb{C}$-submodule of the ambient $\mathrm{SL}_{3} \mathbb{C}$-module under consideration. Note also that $\mathrm{SO}_{3} \mathbb{C} \simeq \mathrm{PSL}_{2} \mathbb{C}$, so we are really back in the much classically studied theory of binary forms. It is not difficult (and fun) to check (12), (13), (14), (15) by hand; let us briefly digress on how this can be done (cf. [Fu-Ha]):

We fix the following notation. Let first $n=2 l+1$ be an odd integer, $\mathfrak{g}=\mathfrak{s l}_{n} \mathbb{C}$ the Lie algebra of $\mathrm{SL}_{n} \mathbb{C}$, and let $\mathfrak{t}_{\mathfrak{g}}$ its standard torus of diagonal matrices of trace 0 , and define the standard weights $\epsilon_{i} \in \mathfrak{t}_{\mathfrak{g}}^{\vee}$, $i=1, \ldots, n$, by $\epsilon_{i}\left(\operatorname{diag}\left(x_{1}, \ldots, x_{n}\right)\right):=x_{i}$. Inside $\mathfrak{g}$ we find $\mathfrak{h}:=\mathfrak{s o}_{n} \mathbb{C}$ defined by

$$
\mathfrak{h}:=\left\{\begin{array}{c}
\left(\begin{array}{ccc}
X & Y & U \\
Z & -X^{t} & V \\
-V^{t} & -U^{t} & 0
\end{array}\right) \mid X, Y, Z \in \mathfrak{g l}_{l} \mathbb{C}, Y^{t}=-Y^{t}, \\
\left.Z=-Z^{t}, U, V \in \mathbb{C}^{l}\right\} .
\end{array}\right.
$$

Then $\mathfrak{t}_{\mathfrak{h}}:=\left\{\operatorname{diag}\left(x_{1}, \ldots, x_{l},-x_{1}, \ldots,-x_{l}, 0\right) \mid x_{i} \in \mathbb{C}\right\}$; by abuse of notation we denote the restrictions of the functions $\epsilon_{i}$ to $\mathfrak{t}_{\mathfrak{h}}$ by the same letters. The fundamental weights of $\mathfrak{g}$ are $\pi_{i}:=\epsilon_{1}+\cdots+\epsilon_{i}$, 
$i=1, \ldots, n-1$, the fundamental weights of $\mathfrak{h}$ are $\omega_{i}:=\epsilon_{1}+\cdots+\epsilon_{i}$, $(1 \leq i \leq l-1)$ and $\omega_{l}:=\left(\epsilon_{1}+\cdots+\epsilon_{l}\right) / 2$. Let $\Lambda_{\mathfrak{g}}$ and $\Lambda_{\mathfrak{h}}$ be the corresponding weight lattices. $\Lambda_{\mathfrak{g}}^{+}$and $\Lambda_{\mathfrak{h}}^{+}$are the dominant weights. For $\mathfrak{g}$ (and similarly for $\mathfrak{h}$ ) an irreducible representation $V(\lambda)$ for $\lambda \in$ $\Lambda_{\mathfrak{g}}^{+}$comes with its formal character

$$
\operatorname{ch}_{\lambda}:=\sum_{\mu \in \Pi(\lambda)} m_{\lambda}(\mu) e(\mu) \in \mathbb{Z}\left[\Lambda_{\mathfrak{g}}\right],
$$

an element of the group algebra $\mathbb{Z}\left[\Lambda_{\mathfrak{g}}\right]$ generated by the symbols $e(\lambda)$ for $\lambda \in \Lambda_{\mathfrak{g}}$, where $\Pi(\lambda)$ means the weights of $V(\lambda)$, and $m_{\lambda}(\mu)$ is the dimension of the weight space corresponding to $\mu$ in $V(\lambda)$. We have a formal character $\mathrm{ch}_{V}$ for any finite-dimensional $\mathfrak{g}$-module $V=$ $V\left(\lambda_{1}\right) \oplus \cdots \oplus V\left(\lambda_{t}\right), \lambda_{1}, \ldots, \lambda_{t} \in \Lambda_{\mathfrak{g}}^{+}$defined by

$$
\operatorname{ch}_{V}:=\sum_{i=1}^{t} \operatorname{ch}_{\lambda_{i}} .
$$

The important point is that $V$ (i.e. its irreducible constituents) can be recovered from the formal character $\mathrm{ch}_{V}$, meaning that in $\mathbb{Z}\left[\Lambda_{\mathfrak{g}}\right]$ we can write $\operatorname{ch}_{V}$ uniquely as a $\mathbb{Z}$-linear combination of characters corresponding to dominant weights $\lambda \in \Lambda_{\mathfrak{g}}^{+}$.

We go back to the case $l=1, n=3$. We have $\mathfrak{h}=\mathfrak{s o}_{3} \mathbb{C}=\mathfrak{s l}_{2} \mathbb{C}$. The character $\operatorname{ch}_{V(a)}$ of the irreducible $\mathfrak{s o}_{3} \mathbb{C}$-module $V(a):=V\left(a \omega_{1}\right)$ is not hard: The weights of $V(a)$ are

$$
-a \omega_{1},(-a+2) \omega_{1}, \ldots,(a-2) \omega_{1}, a \omega_{1}
$$

(all multiplicities are 1). It remains to understand the weights and their multiplicities in the irreducible $\mathfrak{g}=\mathfrak{s l}_{3} \mathbb{C}$-module $V(a, b):=V\left(a \pi_{1}+\right.$ $\left.b \pi_{2}\right)$. In fact noting that $\pi_{1}$ restricted to the diagonal torus of $\mathfrak{s o}_{3} \mathbb{C}$ above is $2 \omega_{1}$, and the restriction of $\pi_{2}$ is 0 , we see that, once we know the formal character of $V(a, b)$ as $\mathfrak{s l}_{3} \mathbb{C}$-module, we simply substitute $2 \omega_{1}$ for $\pi_{1}$ and 0 for $\pi_{2}$ in the result and obtain in this way the formal character of the $\mathfrak{s o}_{3} \mathbb{C}$-module $V(a, b)$, and hence its decomposition into irreducible constituents as $\mathfrak{s o}_{3} \mathbb{C}$-module.

Let us assume $a \geq b$ (otherwise pass to the dual representation); we describe the weights and their multiplicities of the $\mathfrak{s l}_{3} \mathbb{C}$-module $V(a, b)$ following [Fu-Ha], p. 175ff.: Imagine a plane with a chosen origin from which we draw two vectors of unit length, representing $\pi_{1}$ and $\pi_{2}$, such that the angle measured counterclockwise from $\pi_{1}$ to $\pi_{2}$ is $60^{\circ}$. Thus the points of the lattice spanned by $\pi_{1}, \pi_{2}$ are the vertices of a set of equilateral congruent triangles which gives a tiling of the plane.

The weights of $V(a, b)$ are the lattice points which lie on the edges of 
a sequence of $b$ (not necessarily regular) hexagons $H_{i}$ with vertices at lattice points, $i=0, \ldots, b-1$, and a sequence of $[(a-b) / 3]+1$ triangles $T_{j}, j=0, \ldots,[(a-b) / 3]$. The $H_{i}$ and $T_{j}$ are concentric around the origin, and $H_{i}$ has one vertex at $(a-i) \pi_{1}+(b-i) \pi_{2}, T_{j}$ has one vertex at the point $(a-b-3 j) \pi_{1}$, and $H_{i}$ and $T_{j}$ are otherwise determined by the condition that the lines through $\pi_{1}, \pi_{2}, \pi_{2}-\pi_{1}$ are axes of symmetry for them, i.e. they are preserved by the reflections in these lines (one should make a picture now).

The multiplicities of the weights obtained in this way are as follows: Weights lying on $H_{i}$ have multiplicity $i+1$, and weights lying on one of the $T_{j}$ have multiplicity $b$. This completely determines the formal character of $V(a, b)$.

Let us look at $V(2,2)$ for example. Here we get three concentric regular hexagons (one of them is degenerate and consists of the origin alone). The weights are thus:

$$
\begin{gathered}
2 \pi_{1}+2 \pi_{2}, 3 \pi_{2},-2 \pi_{1}+4 \pi_{2},-3 \pi_{1}+3 \pi_{2},-4 \pi_{1}+2 \pi_{2},-3 \pi_{1}, \\
\quad-2 \pi_{1}-2 \pi_{2},-3 \pi_{2}, 2 \pi_{1}-4 \pi_{2}, 3 \pi_{1}-3 \pi_{2}, 4 \pi_{1}-2 \pi_{2}, 3 \pi_{1}
\end{gathered}
$$

(these are the ones on the outer hexagon, read counterclockwise, and have multiplicity one),

$$
\pi_{1}+\pi_{2},-\pi_{1}+2 \pi_{2},-2 \pi_{1}+\pi_{2},-\pi_{1}-\pi_{2}, \pi_{1}-2 \pi_{2}, 2 \pi_{1}-\pi_{2}
$$

(these lie on the middle hexagon and have multiplicity two), and finally there is 0 with multiplicity 3 corresponding to the origin. Consequently, the formal character of $V(2,2)$ as a representation of $\mathfrak{s o}_{3} \mathbb{C}$ is

$$
\begin{aligned}
e\left(-8 \omega_{1}\right) & +2 e\left(-6 \omega_{1}\right)+4 e\left(-4 \omega_{1}\right)+4 e\left(-2 \omega_{1}\right)+5 e\left(0 \omega_{1}\right), \\
& +4 e\left(2 \omega_{1}\right)+4 e\left(4 \omega_{1}\right)+2 e\left(6 \omega_{1}\right)+e\left(8 \omega_{1}\right)
\end{aligned}
$$

which is equal to $\mathrm{ch}_{V(8)}+\operatorname{ch}_{V(6)}+2 \mathrm{ch}_{V(4)}+\mathrm{ch}_{V(0)}$. This proves (12), and (13), (14) and (15) are similar.

We resume the discussion of the main content of subsection 2.4. Before stating the main theorem, we collect some preliminary facts in the following lemma.

Lemma 2.4.2. (1) The following deccomposition of $L \subset V(2,2)$ as $\mathrm{SO}_{3} \mathbb{C}$-subspace of $V(2,2)$ holds (possibly after interchanging the roles of $V(2,2)_{4}$ and $\left.V(2,2)_{4}^{\prime}\right)$ :

$$
L=V(2,2)_{8} \oplus V(2,2)_{4} \oplus V(2,2)_{0} .
$$

(2) The map $\epsilon\left(\cdot, h_{0}\right): V(0,4) \rightarrow V(2,2)$ is an $\mathrm{SO}_{3}$-equivariant isomorphism onto $L$.

(3) Putting $Y:=\epsilon\left(\cdot, h_{0}\right)^{-1}(Z) \subset V(0,4)$, we have $h_{0}^{2} \in Y$. 
(4) One has $\Gamma(V(0,4)) \subset V(1,1)_{4} \subset V(1,1)$, and the inclusion $Y \subset \Gamma^{-1}(0)$ holds.

Proof. (1): Using the explicit form of $\Phi$ one calculates that the dimension of the image of the $\mathrm{SO}_{3} \mathbb{C}$-equivariant map $\Phi\left(\cdot, h_{0}\right): V(2,2) \rightarrow$ $V(2,1)$ is 12 . Thus, in view of the decomposition (13) of $V(2,1)$ as $\mathrm{SO}_{3} \mathbb{C}$-representation, we must have $\Phi\left(V(2,2), h_{0}\right)=V(2,1)_{6} \oplus$ $V(2,1)_{4}$. Since

$$
\operatorname{dim} V(a, b)=\frac{1}{2}(a+1)(b+1)(a+b+2),
$$

the dimension of $V(2,2)$ is 27 and the kernel $L$ of $\Phi\left(\cdot, h_{0}\right)$ has dimension 15 ; in fact, $V(2,2)_{8}, V(2,2)_{0}$ and (after possibly exchanging $V(2,2)_{4}$ and $\left.V(2,2)_{4}^{\prime}\right) V(2,2)_{4}$ must all be in the kernel, since these representations do not appear in the decomposition of the image.

(2): Using the explicit form of $\epsilon$ given in Appendix A (66), one calculates that the dimension of the image of $\epsilon\left(\cdot, h_{0}\right)$ is 15 whence this linear map is injective. Moreover, its image is contained in $L$, hence equals $L$, because the map $V(0,4) \times V(0,2) \rightarrow V(2,1)$ given by $(f, g) \mapsto$ $\Phi(\epsilon(f, g), g)$ is identically zero since there is no $V(2,1)$ in the decomposition of $V(0,4) \otimes \operatorname{Sym}^{2} V(0,2)$.

(3): As we saw in theorem $2.3 .3(1), \mathbb{C} h_{0}^{2} \in X$, and we have $0 \neq$ $\Psi\left(h_{0}^{2}\right) \in Z$. From the decomposition (12), we get, $\Psi\left(h_{0}^{2}\right)$ being invariant, $\left\langle\Psi\left(h_{0}^{2}\right)\right\rangle_{\mathbb{C}}=L^{\mathrm{SO}_{3} \mathbb{C}}$. By the decomposition (15), we get that the preimage under $\epsilon\left(\cdot, h_{0}\right)$ of $\Psi\left(h_{0}^{2}\right)$ spans the $\mathrm{SO}_{3} \mathbb{C}$-invariants $V(0,4)_{0}$ which are thus in $Y$. So in particular, $h_{0}^{2} \in Y$.

(4): The first part is straightforward: Just decompose $\operatorname{Sym}^{2} V(0,4)$ as $\mathrm{SO}_{3} \mathbb{C}$-module by the methods explained above, and check that it does not contain any $\mathrm{SO}_{3} \mathbb{C}$-submodule the highest weight of which has numerical label 2 (this suffices by (14)). The second statement of (4) follows from the observation that the map $\zeta: V(0,4) \times V(0,2) \rightarrow V(1,1)$ (Def. 2.4.1 (4)) factors:

$$
c \cdot \zeta=\tilde{\gamma} \circ \epsilon, c \in \mathbb{C}^{*},
$$

where $\tilde{\gamma}: V(2,2) \rightarrow V(1,1)$ is the unique (up to nonzero scalar) non-trivial $\mathrm{SL}_{3} \mathbb{C}$-equivariant map which is homogeneous of degree 2. This is because $V(1,1)$ occurs in the decomposition of $\operatorname{Sym}^{2} V(0,4) \otimes$ Sym $^{2} V(0,2)$ with multiplicity one, and $\tilde{\gamma} \circ \epsilon$ is not identically zero, as follows from the explicit form of these maps (cf. Appendix A, (66), (68)). Thus, defining $\tilde{\Gamma}: V(0,4) \rightarrow V(1,1)$ by $\tilde{\Gamma}(\cdot):=(\tilde{\gamma} \circ \epsilon)\left(\cdot, h_{0}\right)$ (which thus differs from $\Gamma$ just by a nonzero scalar), we must show $\tilde{\Gamma}(Y)=0$. But recalling the definitions of $Y, \tilde{\Gamma}$ and $Z$ (Def. 2.4.1 (1)), it suffices to show that $\tilde{\gamma} \circ \Psi$ is identically zero; the latter is true since 
it is an $\mathrm{SL}_{3} \mathbb{C}$-equivariant map from $V(0,4)$ to $V(1,1)$, homogeneous of degree 6 , but $\operatorname{Sym}^{6} V(0,4)$ does not contain $V(1,1)$. This proves (4).

Let us now pass from $\mathrm{SO}_{3} \mathbb{C}$ to the $\mathrm{PSL}_{2} \mathbb{C}$-picture and denote by $V(d)$ the space of binary forms of degree $d$ in the variables $z_{1}, z_{2}$. This is of course consistent with our previous notation since, under the isomorphism $\mathfrak{s o}_{3} \mathbb{C} \simeq \mathfrak{s l}_{2} \mathbb{C}, V(d)$ is just the irreducible $\mathfrak{s o}_{3} \mathbb{C}$-module the highest weight of which has numerical label $d$; since we consider $\mathrm{PSL}_{2} \mathbb{C}$-representations, $d$ is always even.

We will fix a covering $\mathrm{SL}_{2} \mathbb{C} \rightarrow \mathrm{SO}_{3} \mathbb{C}$ and thus an isomorphism $\mathrm{PSL}_{2} \mathbb{C}$ $\simeq \mathrm{SO}_{3} \mathbb{C}$, and we will fix isomorphisms $\delta_{1}: V(0) \oplus V(4) \oplus V(8) \rightarrow$ $V(0,4)$ and $\delta_{2}: V(4) \rightarrow V(1,1)_{4}$ such that $(1,0,0)$ maps to $h_{0}^{2}$ under $\delta_{1}$ and both $\delta_{1}$ and $\delta_{2}$ are equivariant with respect to the isomorphism $\mathrm{PSL}_{2} \mathbb{C} \simeq \mathrm{SO}_{3} \mathbb{C}$; we will discuss in a moment how this is done, but for now this is not important. Look at the diagram

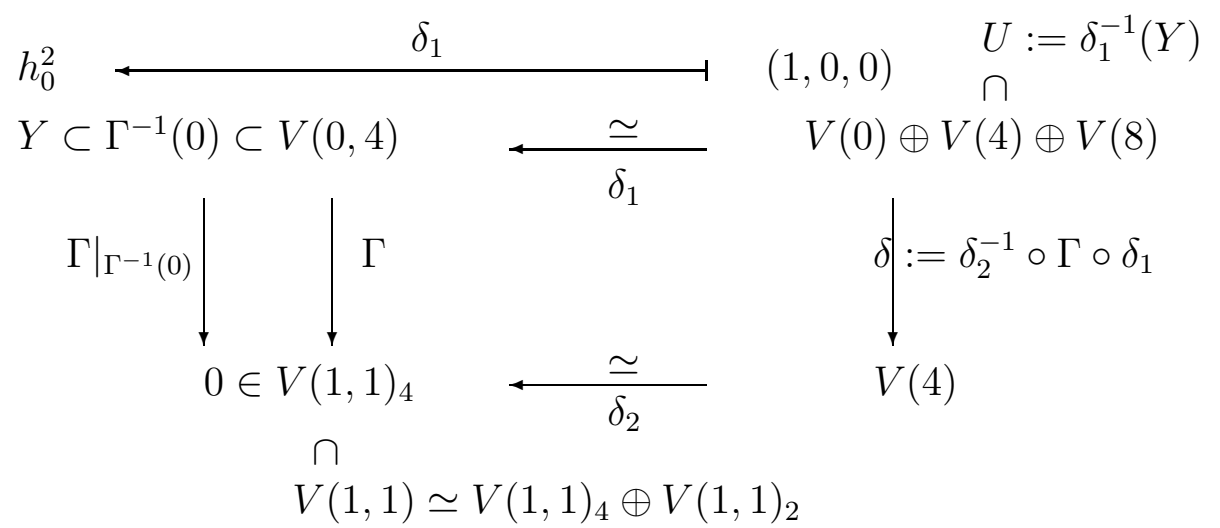

By part (4) of lemma 2.4.2, we have $\delta^{-1}(0) \supset U$, and by part (3) of the same lemma, $(1,0,0) \in U$. Moreover, recalling our construction of $X$ in theorem 2.3.3, we see that $\operatorname{dim} X=\operatorname{dim} \mathbb{P} V(0,4)-\operatorname{dim} \mathbb{P} V(0,2)=$ $14-5=9$, whence, chasing through the definitions of $Z, Y, U$, we get $\operatorname{dim} U=10$. But the explicit form of $\delta$ (we will see this in a moment) allows us to conclude, by explicit calculation of the rank of the differential of $\delta$ at the invariant point $(1,0,0)$, that $\operatorname{dim} T_{(1,0,0)} U=10$, whence $T_{(1,0,0)} U=V(0) \oplus V(8)$. Therefore, as $U$ is irreducible, it is the unique component of the (possibly reducible) variety $\delta^{-1}(0)$ passing through $(1,0,0)$. Moreover, it is clear the condition $\{\delta=0\}$ amounts to 5 quadratic equations! We have proven 
Theorem 2.4.3. There is an isomorphism

$$
\mathbb{C}(\mathbb{P} V(0,4))^{\mathrm{SL}_{3} \mathbb{C}} \simeq \mathbb{C}(U)^{\mathrm{PSL}_{2} \mathbb{C} \times \mathbb{C}^{*}}
$$

where

$$
\delta: V(0) \oplus V(4) \oplus V(8) \rightarrow V(4)
$$

is $\mathrm{PSL}_{2}$-equivariant and homogeneous of degree 2 , and $U$ is the unique irreducible component of $\delta^{-1}(0)$ passing through $(1,0,0)$. Moreover, $\operatorname{dim} U=10$ and $T_{(1,0,0)} U=V(0) \oplus V(8)$.

We close this section by describing the explicit form of the covering $\mathrm{SL}_{2} \mathbb{C} \rightarrow \mathrm{SO}_{3} \mathbb{C}$ and the maps $\delta_{1}, \delta_{2}$, and by making some remarks on transvectants and the final formula for the map $\delta$.

Let $e_{1}, e_{2}, e_{3}$ be the standard basis in $\mathbb{C}^{3}$, and denote by $x_{1}, x_{2}, x_{3}$ the dual basis in $\left(\mathbb{C}^{3}\right)^{\vee}$. In this notation, $h_{0}^{2}=x_{1} x_{3}-x_{2}^{2}$. We may view the $x^{\prime} s$ as coordinates on $\mathbb{C}^{3}$ and identify $\mathbb{C}^{3}$ with the Lie algebra $\mathfrak{s l}_{2} \mathbb{C}$ by assigning to $\left(x_{1}, x_{2}, x_{3}\right)$ the matrix

$$
X=\left(\begin{array}{ll}
x_{2} & -x_{1} \\
x_{3} & -x_{2}
\end{array}\right) \in \mathfrak{s l}_{2} \mathbb{C}
$$

Consider the adjoint representation $\mathrm{Ad}$ of $\mathrm{SL}_{2} \mathbb{C}$ on $\mathfrak{s l}_{2} \mathbb{C}$. Clearly, for $X \in \mathfrak{s l}_{2} \mathbb{C}, A \in \mathrm{SL}_{2} \mathbb{C}$, the $\operatorname{map} \operatorname{Ad}(A): X \mapsto A X A^{-1}$ preserves the determinant of $X$, which is just our $h_{0}$; the kernel of Ad is the center $\{ \pm 1\}$ of $\mathrm{SL}_{2} \mathbb{C}$, and since $\mathrm{SL}_{2} \mathbb{C}$ is connected, the image of Ad is $\mathrm{SO}_{3} \mathbb{C}$. This is how we fix the isomorphism $\mathrm{PSL}_{2} \mathbb{C} \simeq \mathrm{SO}_{3} \mathbb{C}$ explicitly, and how we view $\mathrm{SO}_{3} \mathbb{C}$ as a subgroup of $\mathrm{SL}_{3} \mathbb{C}$. Note that the induced isomorphism $\mathfrak{s l}_{2} \mathbb{C} \rightarrow \mathfrak{s o}_{3} \mathbb{C}$ on the Lie algebra level can be described as follows:

$$
\begin{gathered}
e:=\left(\begin{array}{ll}
0 & 1 \\
0 & 0
\end{array}\right) \mapsto\left(\begin{array}{lll}
0 & 2 & 0 \\
0 & 0 & 1 \\
0 & 0 & 0
\end{array}\right), \\
f:=\left(\begin{array}{cc}
0 & 0 \\
1 & 0
\end{array}\right) \mapsto\left(\begin{array}{lll}
0 & 0 & 0 \\
1 & 0 & 0 \\
0 & 2 & 0
\end{array}\right), \\
h:=\left(\begin{array}{cc}
1 & 0 \\
0 & -1
\end{array}\right) \mapsto\left(\begin{array}{ccc}
2 & 0 & 0 \\
0 & 0 & 0 \\
0 & 0 & -2
\end{array}\right)
\end{gathered}
$$


(where we view $\mathfrak{s o}_{3} \mathbb{C}$ as a subalgebra of $\mathfrak{s l}_{3} \mathbb{C}$ in a way consistent with the inclusion on the group level described above). For example,

$$
\begin{gathered}
\operatorname{ad}\left(\left(\begin{array}{ll}
0 & 1 \\
0 & 0
\end{array}\right)\right)(X)=\left(\begin{array}{ll}
0 & 1 \\
0 & 0
\end{array}\right)\left(\begin{array}{ll}
x_{2} & -x_{1} \\
x_{3} & -x_{2}
\end{array}\right) \\
-\left(\begin{array}{ll}
x_{2} & -x_{1} \\
x_{3} & -x_{2}
\end{array}\right)\left(\begin{array}{ll}
0 & 1 \\
0 & 0
\end{array}\right)=\left(\begin{array}{cc}
x_{3} & -2 x_{1} \\
0 & -x_{3}
\end{array}\right),
\end{gathered}
$$

SO

$$
\left(\begin{array}{l}
x_{1} \\
x_{2} \\
x_{3}
\end{array}\right) \mapsto\left(\begin{array}{lll}
0 & 2 & 0 \\
0 & 0 & 1 \\
0 & 0 & 0
\end{array}\right)\left(\begin{array}{l}
x_{1} \\
x_{2} \\
x_{3}
\end{array}\right)
$$

To give the isomorphism $\delta_{1}: V(0) \oplus V(4) \oplus V(8) \rightarrow V(0,4)$ explicitly, we just have to find highest weight vectors inside $V(0), V(4), V(8)$ and corresponding highest weight vectors inside $V(0,4)$. For example, $h$ acts on $z_{2}^{4} \in V(4)$ by multiplication by 4 , and $z_{2}^{4}$ is killed by $e$, so this is a highest weight vector inside $V(4)$. But if we compute

$$
\begin{gathered}
h \cdot\left(x_{1} x_{3}^{3}-x_{2}^{2} x_{3}^{2}\right)=\left(h \cdot x_{1}\right) x_{3}^{3}+3 x_{1}\left(h \cdot x_{3}\right) x_{3}^{2}-2\left(h \cdot x_{2}\right) x_{2} x_{3}^{2} \\
-2 x_{2}^{2}\left(h \cdot x_{3}\right) x_{3}=\left(-2 x_{1}\right) x_{3}^{3}+3 x_{1}\left(2 x_{3}\right) x_{3}^{2}-2 \cdot 0 \cdot x_{2} x_{3}^{2} \\
-2 x_{2}^{2}\left(2 x_{3}\right) x_{3}=4\left(x_{1} x_{3}^{3}-x_{2}^{2} x_{3}^{2}\right) \text { and } \\
e \cdot\left(x_{1} x_{3}^{3}-x_{2}^{2} x_{3}^{2}\right)=\left(e \cdot x_{1}\right) x_{3}^{3}+3 x_{1}\left(e \cdot x_{3}\right) x_{3}^{2}-2\left(e \cdot x_{2}\right) x_{2} x_{3}^{2} \\
-2 x_{2}^{2}\left(e \cdot x_{3}\right) x_{3}=\left(-2 x_{2}\right) \cdot x_{3}^{3}+3 x_{1} \cdot 0 \cdot x_{3}^{2}-2\left(-x_{3}\right) x_{2} x_{3}^{2} \\
-2 x_{2}^{2} \cdot 0 \cdot x_{3}=0
\end{gathered}
$$

(use (18) and remark that the $x$ 's are dual variables, so we have to use the dual action), then we find that a corresponding highest weight vector for the submodule of $V(0,4)$ isomorphic to $V(4)$ is $x_{1} x_{3}^{3}-x_{2}^{2} x_{3}^{2}$. Proceeding in this way, we see that we can define $\delta_{1}$ uniquely by the requirements:

$$
\delta_{1}: 1 \mapsto h_{0}^{2}, z_{2}^{4} \mapsto x_{1} x_{3}^{3}-x_{2}^{2} x_{3}^{2}, z_{2}^{8} \mapsto x_{3}^{4},
$$

and using the Lie algebra action and linearity, we can compute the values of $\delta_{1}$ on a set of basis vectors in $V(0) \oplus V(4) \oplus V(8)$.

To write down $\delta_{2}$ explicitly, remark that $V(1,1)$ may be viewed as the $\mathrm{SL}_{3} \mathbb{C}$-submodule of $\mathbb{C}^{3} \otimes\left(\mathbb{C}^{3}\right)^{\vee}$ consisting of those tensors that are annihilated by

$$
\Delta:=\frac{\partial}{\partial e_{1}} \otimes \frac{\partial}{\partial x_{1}}+\frac{\partial}{\partial e_{2}} \otimes \frac{\partial}{\partial x_{2}}+\frac{\partial}{\partial e_{3}} \otimes \frac{\partial}{\partial x_{3}} .
$$

We take again our highest weight vector $z_{2}^{4} \in V(4)$, and all we have to do is to find a vector in $\mathbb{C}^{3} \otimes\left(\mathbb{C}^{3}\right)^{\vee}$ on which $h$ acts by multiplication 
by 4 and which is annihilated by $e$ and $\Delta$. Indeed, $e_{1} x_{3}$ is one such. Thus we define $\delta_{2}$ by

$$
\delta_{2}: z_{2}^{4} \mapsto e_{1} x_{3}
$$

Then it is easy to compute the values of $\delta_{2}$ on basis elements of $V(4)$ in the same way as for $\delta_{1}$.

Let us recall the classical notion of transvectants " Überschiebung " in German). Let $d_{1}, d_{2}, n$ be nonnegative integers such that $0 \leq n \leq$ $\min \left(d_{1}, d_{2}\right)$. For $f \in V\left(d_{1}\right)$ and $g \in V\left(d_{2}\right)$ one puts

$$
\psi_{n}(f, g):=\frac{\left(d_{1}-n\right) !}{d_{1} !} \frac{\left(d_{2}-n\right) !}{d_{2} !} \sum_{i=0}^{n}(-1)^{i}\left(\begin{array}{c}
n \\
i
\end{array}\right) \frac{\partial^{n} f}{\partial z_{1}^{n-i} \partial z_{2}^{i}} \frac{\partial^{n} g}{\partial z_{1}^{i} \partial z_{2}^{n-i}}
$$

(cf. [B-S], p. 122). The map $(f, g) \mapsto \psi_{n}(f, g)$ is a bilinear and $\mathrm{SL}_{2} \mathbb{C}$ equivariant map from $V\left(d_{1}\right) \times V\left(d_{2}\right)$ onto $V\left(d_{1}+d_{2}-2 n\right)$. The map

$$
\begin{aligned}
V\left(d_{1}\right) \otimes V\left(d_{2}\right) & \rightarrow \bigoplus_{n=0}^{\min \left(d_{1}, d_{2}\right)} V\left(d_{1}+d_{2}-2 n\right) \\
(f, g) & \mapsto \sum_{n=0}^{\min \left(d_{1}, d_{2}\right)} \psi_{n}(f, g)
\end{aligned}
$$

is an isomorphism of $\mathrm{SL}_{2} \mathbb{C}$-modules ("Clebsch-Gordan decomposition"). Thus transvectants make the decomposition of $V\left(d_{1}\right) \otimes V\left(d_{2}\right)$ into irreducibles explicit; a similar result for $\mathrm{SL}_{3} \mathbb{C}$-representations would be very important in several areas of computational invariant theory and also for the rationality question for moduli spaces of plane curves, but is apparently unknown.

The explicit form of $\delta$ that results from the computations is then

$$
\begin{aligned}
\delta\left(f_{0}, f_{4}, f_{8}\right) & =-\frac{6}{1225} \psi_{6}\left(f_{8}, f_{8}\right)+\frac{1}{840} \psi_{4}\left(f_{8}, f_{4}\right) \\
+ & \frac{11}{54} \psi_{2}\left(f_{4}, f_{4}\right)-\frac{7}{36} f_{4} f_{0},
\end{aligned}
$$

where $\left(f_{0}, f_{4}, f_{8}\right) \in V(0) \oplus V(4) \oplus V(8)$. Note that the fact that $\delta$ turns out to be such a linear combination of transvectants is no surprise in view of the Clebsch-Gordan decomposition: In fact, $\delta$ may be viewed as a map

$$
\delta^{\prime}:(V(0) \oplus V(4) \oplus V(8)) \otimes(V(0) \oplus V(4) \oplus V(8)) \rightarrow V(4)
$$


and using the fact that $\delta$ is symmetric and collecting only those tensor products in the preceding formula for which $V(4)$ is a subrepresentation, we see that $\delta$ comes from a map

$$
\begin{gathered}
\delta^{\prime \prime}:(V(0) \otimes V(4)) \oplus(V(4) \otimes V(4)) \\
\oplus(V(8) \otimes V(4)) \oplus(V(8) \otimes V(8)) \rightarrow V(4) .
\end{gathered}
$$

Thus it is clear from the beginning that $\delta$ will be a linear combination of $\psi_{6}, \psi_{4}, \psi_{2}, \psi_{0}$ as in formula $(21)$, and the actual coefficients are easily calculated once we know $\delta$ explicitly!

In fact, the next lemma shows that the actual coefficients of the transvectants $\psi_{i}$ 's occurring in $\delta$ are not very important.

Lemma 2.4.4. For $\lambda:=\left(\lambda_{0}, \lambda_{2}, \lambda_{4}, \lambda_{6}\right) \in \mathbb{C}^{4}$ consider the homogeneous of degree $2 \mathrm{PSL}_{2}$-equivariant map

$$
\begin{gathered}
\delta_{\lambda}: V(8) \oplus V(0) \oplus V(4) \rightarrow V(4) \\
f_{8}+f_{0}+f_{4} \mapsto \lambda_{6} \psi_{6}\left(f_{8}, f_{8}\right)+2 \lambda_{4} \psi_{4}\left(f_{8}, f_{4}\right)+\lambda_{2} \psi_{2}\left(f_{4}, f_{4}\right)+2 \lambda_{0} f_{4} f_{0} .
\end{gathered}
$$

Suppose that $\lambda_{0} \neq 0$. Then:

(1) One has $1 \in \delta_{\lambda}^{-1}(0)$ and $T_{1} \delta_{\lambda}^{-1}(0)=V(8) \oplus V(0)$; thus there is a unique irreducible component $U_{\lambda}$ of $\delta_{\lambda}^{-1}(0)$ passing through 1 on which 1 is a smooth point.

(2) If furthermore $\lambda \in\left(\mathbb{C}^{*}\right)^{4}$, then $\mathbb{P} U_{\lambda}$ is $\mathrm{PSL}_{2} \mathbb{C}$-equivariantly isomorphic to $\mathbb{P} U_{(1,6 \epsilon, 1,6)}$ for some $\epsilon \neq 0$ (depending on $\lambda$ ).

Proof. Part (1) is a straightforward calculation, and for part (2) we choose complex numbers $\mu_{0}, \mu_{4}, \mu_{8}$ with the properties $6 \mu_{8}^{2}=\lambda_{6}$, $\mu_{4} \mu_{8}=\lambda_{4}, \mu_{0} \mu_{4}=\lambda_{0}$, and compute $\epsilon$ from $6 \epsilon \mu_{4}^{2}=\lambda_{2}$. Then the map from $\mathbb{P} U_{\lambda}$ to $\mathbb{P} U_{(1,6 \epsilon, 1,6)}$ given by sending $\left[f_{0}+f_{4}+f_{8}\right]$ to $\left[\mu_{0} f_{0}+\mu_{4} f_{4}+\right.$ $\left.\mu_{8} f_{8}\right]$ gives the desired isomorphism.

In the next section we will see that for any $\epsilon \neq 0$, the $\mathrm{PSL}_{2} \mathbb{C}$ quotient of $\mathbb{P} U_{(1,6 \epsilon, 1,6)}$ is rational, and so the same holds for $\mathbb{P} U_{\lambda}$ for any $\lambda \in\left(\mathbb{C}^{*}\right)^{4}$; note however that the reduction step in lemma 2.4.4 (2) just simplifies the subsequent calculations, but is otherwise not substantial.

\section{FURTHER SECTIONS AND INNER PROJECTIONS}

3.1. Binary quartics again and a $\left(\mathrm{PSL}_{2} \mathbb{C}, \mathfrak{S}_{4}\right)$-section. All the subsequent constructions and calculations depend very much on the geometry of the $\mathrm{PSL}_{2} \mathbb{C}$-action on the module $V(4)$. In fact, the first main point in the proof that $\mathbb{P} U_{\lambda} / \mathrm{PSL}_{2} \mathbb{C}$ is rational will be the construction of a $\left(\mathrm{PSL}_{2} \mathbb{C}, \mathfrak{S}_{4}\right)$-section of this variety $\left(\mathfrak{S}_{4}\right.$ being the group of permutations of 4 elements); this is done by using proposition 2.1.2 
(2) for the projection of $V(8) \oplus V(0) \oplus V(4)$ to $V(4)$ and producing such a section for $V(4)$ via the concept of stabilizer in general position which we recall next.

Definition 3.1.1. Let $G$ be a linear algebraic group $G$ acting on an irreducible variety $X$. A stabilizer in general position (s.g.p.) for the action of $G$ on $X$ is a subgroup $H$ of $G$ such that the stabilizer of a general point in $X$ is conjugate to $H$ in $G$.

An s.g.p. (if it exists) is well-defined to within conjugacy, but it need not exist in general; however, for the action of a reductive group $G$ on an irreducible smooth affine variety, an s.g.p. always exists by results of Richardson and Luna (cf. [Po-Vi], §7).

Proposition 3.1.2. For the action of $\mathrm{PSL}_{2} \mathbb{C}$ on $V(4)$, an s.g.p. is given by the subgroup $H$ generated by

$$
\omega:=\left[\left(\begin{array}{cc}
0 & 1 \\
-1 & 0
\end{array}\right)\right] \text { and } \rho:=\left[\left(\begin{array}{cc}
i & 0 \\
0 & -i
\end{array}\right)\right] .
$$

$H$ is isomorphic to the Klein four-group $\mathfrak{V}_{4} \simeq \mathbb{Z} / 2 \mathbb{Z} \oplus \mathbb{Z} / 2 \mathbb{Z}$ and its normalizer $N(H)$ in $\mathrm{PSL}_{2} \mathbb{C}$ is isomorphic to $\mathfrak{S}_{4}$; one has $N(H) / H \simeq$ $\mathfrak{S}_{3}$.

More explicitly, $N(H)=\langle\tau, \sigma\rangle$, where, putting $\theta:=\exp (2 \pi i / 8)$, one has

$$
\tau:=\left[\left(\begin{array}{cc}
\theta^{-1} & 0 \\
0 & \theta
\end{array}\right)\right], \quad \sigma:=\left[\frac{1}{\sqrt{2}}\left(\begin{array}{cc}
\theta^{3} & \theta^{7} \\
\theta^{5} & \theta^{5}
\end{array}\right)\right] .
$$

Proof. We will give a geometric proof due to Bogomolov (Bog1, p.18). A general homogeneous degree 4 binary form $f \in V(4)$ determines a set of 4 points $\Sigma \subset \mathbb{P}^{1}$; the double cover of $\mathbb{P}^{1}$ with branch points $\Sigma$ is an elliptic curve; it is acted on by its subgroup of 2-torsion points $H_{f} \simeq \mathbb{Z} / 2 \mathbb{Z} \oplus \mathbb{Z} / 2 \mathbb{Z}$, and this action commutes with the sheet exchange map, hence descends to an action of $H_{f}$ on $\mathbb{P}^{1}$ which preserves the point set $\Sigma$ and thus the polynomial $f$; in general $H_{f}$ will be the full automorphism group of the point set $\Sigma$ since a general elliptic curve does not have complex multiplication.

Let us see that $H_{f}$ is conjugate to $H: H_{f}$ is generated by two commuting reflections $\gamma_{1}, \gamma_{2}$ acting on the Riemann sphere $\mathbb{P}^{1}$ (with two fixed points each). By applying a suitable projectivity, we see that $H_{f}$ is conjugate to $\left\langle\omega, \gamma_{2}^{\prime}\right\rangle$ where $\gamma_{2}^{\prime}$ is another reflection commuting with $\omega$; thus $\omega$ interchanges the fixed points of $\gamma_{2}^{\prime}$ and also the fixed points of $\rho$ : Thus if we change coordinates via a suitable dilation (a projectivity preserving the fixed points of $\omega), \gamma_{2}^{\prime}$ goes over to $\rho$, and thus $H_{f}$ is conjugate to $H$. 
One computes that $\sigma$ and $\tau$ normalize $H$; in fact, $\sigma^{-1} \omega \sigma=\rho, \sigma^{-1} \rho \sigma=$ $\omega \rho$, and $\tau^{-1} \omega \tau=\omega \rho, \tau^{-1} \rho \tau=\rho$. Moreover, $\tau$ has order 4 and $\sigma$ order $3,(\tau \sigma)^{2}=1$, thus one has the relations

$$
\tau^{4}=\sigma^{3}=(\tau \sigma)^{2}=1 .
$$

It is known that $\mathfrak{S}_{4}$ is the group on generators $R, S$ with relations $R^{4}=S^{2}=(R S)^{3}=1$; mapping $R \mapsto \tau^{-1}, S \mapsto \tau \sigma$, we see that the group $\langle\tau, \sigma\rangle<N(H)$ is a quotient of $\mathfrak{S}_{4}$; since $\langle\tau, \sigma\rangle$ contains elements of order 4 and order 3 , its order is at least 12 , but since there are no normal subgroups of order 2 in $\mathfrak{S}_{4}, \mathfrak{S}_{4}=\langle\tau, \sigma\rangle$. To finish the proof, it therefore suffices to note that the order of $N(H)$ is at most 24: For this one just has to show that the centralizer of $H$ in $\mathrm{PSL}_{2} \mathbb{C}$ is just $H$, for then $N(H) / H$ is a subgroup of the group of permutations of the three nontrivial elements $H-\{1\}$ in $H$ (in fact equal to it). Elements in $\mathrm{PGL}_{2} \mathbb{C}$ commuting with $\omega$ must be of the form

$$
\left[\left(\begin{array}{cc}
a & b \\
-b & a
\end{array}\right)\right] \text { or }\left[\left(\begin{array}{cc}
a & b \\
b & -a
\end{array}\right)\right] \text {, }
$$

and if these commute also with $\rho$, the elements $1, \omega, \rho, \omega \rho$ are the only possibilities.

Corollary 3.1.3. The variety $\left(V(4)^{H}\right)^{0} \subset V(4)$ consisting of those points whose stabilizer in $\mathrm{PSL}_{2} \mathbb{C}$ is exactly $H$ is a $\left(\mathrm{PSL}_{2} \mathbb{C}, N(H)\right)$ section of $V(4)$.

Proof. The fact that the orbit $\mathrm{PSL}_{2} \mathbb{C} \cdot\left(V(4)^{H}\right)^{0}$ is dense in $V(4)$ follows since a general point in $V(4)$ has stabilizer conjugate to $H$; the assertion $\forall g \in \mathrm{PGL}_{2} \mathbb{C}, \forall x \in\left(V(4)^{H}\right)^{0}: g x \in\left(V(4)^{H}\right)^{0} \Longrightarrow g \in N(H)$ is clear by definition.

Let us recall the representation theory of $N(H)=\mathfrak{S}_{4}$ viewed as the group of permutations of four letters $\{a, b, c, d\}$; the character table is as follows (cf. [Se]).

\begin{tabular}{c|rrrrr} 
& 1 & $(a b)$ & $(a b)(c d)$ & $(a b c)$ & $(a b c d)$ \\
\hline$\chi_{0}$ & 1 & 1 & 1 & 1 & 1 \\
$\epsilon$ & 1 & -1 & 1 & 1 & -1 \\
$\theta$ & 2 & 0 & 2 & -1 & 0 \\
$\psi$ & 3 & 1 & -1 & 0 & -1 \\
$\epsilon \psi$ & 3 & -1 & -1 & 0 & 1
\end{tabular}

$V_{\chi_{0}}$ is the trivial 1-dimensional representation, $V_{\epsilon}$ is the 1-dimensional representation where $\epsilon(g)$ is the sign of the permutation $g ; \mathfrak{S}_{4}=N(H)$ being the semidirect product of $N(H) / H=\mathfrak{S}_{3}$ by the normal subgroup 
$H, V_{\theta}$ is the irreducible two-dimensional representation induced from the representation of $\mathfrak{S}_{3}$ acting on the elements of $\mathbb{C}^{3}$ which satisfy $x+y+z=0$ by permutation of coordinates. $V_{\psi}$ is the extension to $\mathbb{C}^{3}$ of the natural representation of $\mathfrak{S}_{4}$ on $\mathbb{R}^{3}$ as the group of rigid motions stabilizing a regular tetrahedron; finally, $V_{\epsilon \psi}=V_{\epsilon} \otimes V_{\psi}$.

We want to decompose $V(8) \oplus V(0) \oplus V(4)$ as $N(H)$-module; we fix the notation:

$$
\begin{gathered}
a_{0}:=1 ; \quad a_{1}:=z_{1}^{4}+z_{2}^{4}, a_{2}:=6 z_{1}^{2} z_{2}^{2}, a_{3}:=z_{1}^{4}-z_{2}^{4}, \\
a_{4}:=4\left(z_{1}^{3} z_{2}-z_{1} z_{2}^{3}\right), a_{5}:=4\left(z_{1}^{3} z_{2}+z_{1} z_{2}^{3}\right) \\
e_{1}:=28\left(z_{1}^{6} z_{2}^{2}-z_{1}^{2} z_{2}^{6}\right), e_{2}:=56\left(z_{1}^{7} z_{2}+z_{1}^{5} z_{2}^{3}-z_{1}^{3} z_{2}^{5}-z_{1} z_{2}^{7}\right), \\
e_{3}:=56\left(z_{1}^{7} z_{2}-z_{1}^{5} z_{2}^{3}-z_{1}^{3} z_{2}^{5}+z_{1} z_{2}^{7}\right), e_{4}:=z_{1}^{8}-z_{2}^{8} \\
e_{5}:=8\left(z_{1}^{7} z_{2}-7 z_{1}^{5} z_{2}^{3}+7 z_{1}^{3} z_{2}^{5}-z_{1} z_{2}^{7}\right), \\
e_{6}:=8\left(z_{1}^{7} z_{2}+7 z_{1}^{5} z_{2}^{3}+7 z_{1}^{3} z_{2}^{5}+z_{1} z_{2}^{7}\right), \\
e_{7}:=z_{1}^{8}+z_{2}^{8}, e_{8}:=28\left(z_{1}^{6} z_{2}^{2}+z_{1}^{2} z_{2}^{6}\right), e_{9}:=70 z_{1}^{4} z_{2}^{4} .
\end{gathered}
$$

Lemma 3.1.4. One has the following decompositions as $N(H)$-modules:

$$
V(0)=V_{\chi_{0}}, V(4)=V_{\psi} \oplus V_{\theta}, V(8)=V_{\epsilon \psi} \oplus V_{\psi} \oplus V_{\theta} \oplus V_{\chi_{0}} .
$$

More explicitly,

$$
\begin{gathered}
V(0)=\left\langle a_{0}\right\rangle, V(4)=\left\langle a_{3}, a_{4}, a_{5}\right\rangle \oplus\left\langle a_{1}, a_{2}\right\rangle \\
V(8)=\left\langle e_{4}, e_{5}, e_{6}\right\rangle \oplus\left\langle e_{1}, e_{2}, e_{3}\right\rangle \oplus\left\langle e_{8}, 7 e_{7}-e_{9}\right\rangle \oplus\left\langle 5 e_{7}+e_{9}\right\rangle .
\end{gathered}
$$

Here $\left\langle e_{4}, e_{5}, e_{6}\right\rangle$ corresponds to $V_{\epsilon \psi}$ and $\left\langle e_{1}, e_{2}, e_{3}\right\rangle$ corresponds to $V_{\psi}$. Moreover,

$$
V(0)^{H}=\left\langle a_{0}\right\rangle, V(4)^{H}=\left\langle a_{1}, a_{2}\right\rangle, V(8)^{H}=\left\langle e_{7}, e_{8}, e_{9}\right\rangle .
$$

Proof. We will prove (25) first; one observes that quite generally for $k \geq$ $0, V(2 k)^{H}=\left(V(2 k)^{\rho}\right)^{\omega}$ ( $\rho$ and $\omega$ commute) and that the monomials $z_{1}^{j} z_{2}^{2 k-j}, j=0, \ldots, 2 k$, are invariant under $\rho$ if $j+k$ is even, and otherwise anti-invariant, so if $k=2 s$, $\operatorname{dim} V(2 k)^{\rho}=2 s+1$, and if $k=2 s+1, \operatorname{dim} V(2 k)^{\rho}=2 s+1$. Since $\omega$ is also a reflection, we have $2 \operatorname{dim}\left(V(2 k)^{\rho}\right)^{\omega}-\operatorname{dim} V(2 k)^{\rho}=\operatorname{tr}\left(\left.\omega\right|_{V(2 k)^{\rho}}\right)$, and the trace is 1 for $k=2 s$, and -1 for $k=2 s+1$, thus

$$
\operatorname{dim} V(2 k)^{H}=s+1, k=2 s, \quad \operatorname{dim} V(2 k)^{H}=s, k=2 s+1 .
$$

In particular, the $H$-invariants in $V(0), V(4), V(8)$ have the dimensions as claimed in (25), and one checks that the elements given there are indeed invariant.

To prove (23), we use the Clebsch-Gordan formula $V(2 k) \otimes V(2)=$ $V(2 k+2) \oplus V(2 k) \oplus V(2 k-2)$ (cf. (20)) iteratively together with the 
fact that the character of the tensor product of two representations of a finite group is the product of the characters of each of the factors; since $V(2)$ has dimension 3 and $\operatorname{dim} V(2)^{H}=0, V(2)$ is irreducible; the value of the character of the $N(H)$-module $V(2)$ on $\tau$ is 1 , so $V(2)=V_{\epsilon \psi}$. Now $V(2) \otimes V(2)=V(4) \oplus V(2) \oplus V(0)$, and looking at the character table, one checks that

$$
(\epsilon \psi)^{2}=\chi_{0}+(\epsilon \psi)+(\psi)+(\theta) .
$$

This proves the decomposition in (23) for $V(4)$. The decomposition for $V(8)$ is proven similarly (one proves $V(6)=V_{\psi} \oplus V_{\epsilon \psi} \oplus V_{\epsilon}$ first).

The proof of (24) now amounts to checking that the given spaces are invariant under $\sigma$ and $\tau$; finally note that $V_{\epsilon \psi}$ corresponds to $\left\langle e_{4}, e_{5}, e_{6}\right\rangle$ since the value of the character on $\tau$ is 1 .

Recall from Lemma 2.4.4 that we want to prove the rationality of $\left(\mathbb{P} U_{\lambda}\right) / \mathrm{PSL}_{2} \mathbb{C}$ and we can and will always assume in the sequel that $\lambda=(1,6 \epsilon, 1,6)$ for $\epsilon \neq 0$. In view of Lemma 3.1.4 it will be convenient for subsequent calculations to write the map $\delta_{\lambda}: V(8) \oplus V(0) \oplus V(4) \rightarrow$ $V(4)$ in terms of the basis $\left(e_{1}, \ldots, e_{9}, a_{0}, a_{1}, \ldots, a_{5}\right)$ in the source and the basis $\left(a_{1}, \ldots, a_{5}\right)$ in the target. Denote coordinates in $V(8) \oplus V(0) \oplus$ $V(4)$ with respect to the chosen basis by $\left(x_{1}, \ldots, x_{9}, s_{0}, s_{1}, \ldots, s_{5}\right)=$ : $(x, s)$. Then one may write

$$
\delta_{\lambda}(x, s)=\left(\begin{array}{c}
Q_{1}(x, s) \\
\vdots \\
Q_{5}(x, s)
\end{array}\right)
$$

with $Q_{1}(x, s), \ldots, Q_{5}(x, s)$ quadratic in $(x, s)$; their values may be computed using formulas (20), (22), and the definition of $\delta_{\lambda}$ in Lemma 2.4.4, and they can be found in Appendix B.

Theorem 3.1.5. Let $\tilde{\mathcal{Q}}_{\lambda} \subset V(8) \oplus V(0) \oplus V(4)$ be the subvariety defined by the equations $Q_{1}=\cdots=Q_{5}=0, s_{3}=s_{4}=s_{5}=0$. There is exactly one 7-dimensional irreducible component $\mathcal{Q}_{\lambda}$ of $\tilde{\mathcal{Q}}_{\lambda}$ passing through the $N(H)$-invariant point $5 e_{7}+e_{9}$ in $V(8) ; \mathcal{Q}_{\lambda}$ is $N(H)$-invariant and

$$
\mathbb{C}\left(\mathbb{P} U_{\lambda}\right)^{\mathrm{PSL}_{2} \mathbb{C}}=\mathbb{C}\left(\mathbb{P} \mathcal{Q}_{\lambda}\right)^{N(H)} .
$$

Proof. We want to use Proposition 2.1.2, (2).

Note that $5 e_{7}+e_{9} \in U_{\lambda}$ : In fact, $\delta_{\lambda}$ maps the $N(H)$-invariants in $V(8) \oplus V(0) \oplus V(4)$ to the $N(H)$-invariants in $V(4)$ which are 0 . Since $U_{\lambda}$ is the unique irreducible component of $\delta_{\lambda}^{-1}(0)$ passing through $a_{0}=1, U_{\lambda}$ contains the whole plane of invariants $\left\langle a_{0}, 5 e_{7}+e_{9}\right\rangle$.

If we denote by $p: V(8) \oplus V(0) \oplus V(4) \rightarrow V(4)$ the projection, then $\tilde{\mathcal{Q}}_{\lambda}=p^{-1}\left(V(4)^{H}\right) \cap \delta_{\lambda}^{-1}(0)$. Clearly, $\tilde{\mathcal{Q}}_{\lambda}$ is $N(H)$-invariant, and one 
only has to check that $5 e_{7}+e_{9}$ is a nonsingular point on it with tangent space of dimension 7 by direct calculation: Then there is a unique 7-dimensional irreducible component $\mathcal{Q}_{\lambda}$ of $\tilde{\mathcal{Q}}_{\lambda}$ passing through $5 e_{7}+e_{9}$ which is $N(H)$-invariant (since $5 e_{7}+e_{9}$ is an invariant point on it and this point is nonsingular on $\tilde{\mathcal{Q}}_{\lambda}$ ).

It remains to prove (27): $\mathcal{Q}_{\lambda}$ is an irreducible component of $p^{-1}\left(V(4)^{H}\right) \cap$ $U_{\lambda}$ and $\mathcal{Q}_{\lambda}^{0}=\mathcal{Q}_{\lambda} \cap p^{-1}\left(\left(V(4)^{H}\right)^{0}\right)$ is a dense $N(H)$-invariant open subset of $\mathcal{Q}_{\lambda}$ dominating $\left(V(4)^{H}\right)^{0}$. Thus by Proposition 2.1.1 (2),

$$
\mathbb{C}\left(\mathbb{P} U_{\lambda}\right)^{\mathrm{PSL}_{2} \mathbb{C}} \simeq \mathbb{C}\left(\mathbb{P} \mathcal{Q}_{\lambda}^{0}\right)^{N(H)} \simeq \mathbb{C}\left(\mathbb{P} \mathcal{Q}_{\lambda}\right)^{N(H)}
$$

3.2. Dividing by the action of $H$. Next we would like to "divide out" the action by $H$, so that we are left with an invariant theory problem for the group $N(H) / H=\mathfrak{S}_{3}$. Look back at the action of $N(H)$ on $M:=\left\{s_{3}=s_{4}=s_{5}=0\right\} \subset V(8) \oplus V(0) \oplus V(4)$ which is explained in formulas (23), (24); we will adopt the notational convention to denote the irreducible $N(H)$-submodule of $V(8)$ isomorphic to $V_{\psi}$ by $V(8)_{(\psi)}$ and so forth; thus

$$
M=V(0)_{\left(\chi_{0}\right)} \oplus V(4)_{(\theta)} \oplus V(8)_{\left(\chi_{0}\right)} \oplus V(8)_{(\theta)} \oplus V(8)_{(\psi)} \oplus V(8)_{(\epsilon \psi)},
$$

and looking at the character table of $\mathfrak{S}_{4}$, we see that the action of $H$ is nontrivial only on $V(8)_{(\psi)} \oplus V(8)_{(\epsilon \psi)}=\left\langle e_{1}, e_{2}, e_{3}\right\rangle \oplus\left\langle e_{4}, e_{5}, e_{6}\right\rangle$ where $x_{1}, x_{2}, x_{3}$ and $x_{4}, x_{5}, x_{6}$ are coordinates; in terms of these, we have

$$
\begin{gathered}
(\omega)\left(x_{1}, \ldots, x_{6}\right)=\left(-x_{1}, x_{2},-x_{3},-x_{4}, x_{5},-x_{6}\right), \\
(\rho)\left(x_{1}, \ldots, x_{6}\right)=\left(x_{1},-x_{2},-x_{3}, x_{4},-x_{5},-x_{6}\right), \\
(\omega \rho)\left(x_{1}, \ldots, x_{6}\right)=\left(-x_{1},-x_{2}, x_{3},-x_{4},-x_{5}, x_{6}\right),
\end{gathered}
$$

and

$$
\begin{gathered}
\tau\left(x_{1}, \ldots, x_{6}\right)=\left(-x_{1},-i x_{3},-i x_{2}, x_{4},-i x_{6},-i x_{5}\right), \\
\sigma\left(x_{1}, \ldots, x_{6}\right)=\left(4 x_{3},-\frac{i}{4} x_{1}, i x_{2},-8 x_{6},-\frac{i}{8} x_{4},-i x_{5}\right) .
\end{gathered}
$$

Thus we see that the map

$$
\begin{gathered}
\mathbb{P}\left(V(8)_{(\psi)} \oplus V(8)_{(\epsilon \psi)}\right)-\left\{x_{1} x_{2} x_{3}=0\right\} \rightarrow R \times \mathbb{P}^{2}, \\
\left(x_{1}, \ldots, x_{6}\right) \mapsto\left(\left(\frac{x_{4}}{x_{1}}, \frac{x_{5}}{x_{2}}, \frac{x_{6}}{x_{3}}\right),\left(\frac{1}{x_{1}^{2}}: \frac{1}{x_{2}^{2}}: \frac{1}{x_{3}^{2}}\right)\right),
\end{gathered}
$$

where $R=\mathbb{C}^{3}$, is dominant with fibres $H$-orbits, and furthermore $N(H)$-equivariant for a suitable action of $N(H)$ on $R \times \mathbb{P}^{2}$ : In fact, we 
will agree to write

$$
\left(\frac{1}{x_{1}^{2}}: \frac{1}{x_{2}^{2}}: \frac{1}{x_{3}^{2}}\right)=\left(\frac{x_{2} x_{3}}{x_{1}}: \frac{x_{3} x_{1}}{x_{2}}: \frac{x_{1} x_{2}}{x_{3}}\right)
$$

and remark that the subspaces

$$
R=\left\langle\frac{x_{4}}{x_{1}}, \frac{x_{5}}{x_{2}}, \frac{x_{6}}{x_{3}}\right\rangle, \quad T:=\left\langle\frac{x_{2} x_{3}}{x_{1}}, \frac{x_{3} x_{1}}{x_{2}}, \frac{x_{1} x_{2}}{x_{3}}\right\rangle
$$

of the field of fractions of $\mathbb{C}\left[V(8)_{(\psi)} \oplus V(8)_{(\epsilon \psi)}\right]$ are invariant under $\sigma$ and $\tau$ (thus $\mathbb{P}^{2}=\mathbb{P}(T)$ ). If we denote the coordinates with respect to the basis vectors in $R$ resp. $T$ given above by $r_{1}, r_{2}, r_{3}$ resp. $y_{1}, y_{2}, y_{3}$, then the actions of $\tau$ and $\sigma$ are described by

$$
\begin{gathered}
\tau\left(r_{1}, r_{2}, r_{3}\right)=\left(-r_{1}, r_{3}, r_{2}\right), \sigma\left(r_{1}, r_{2}, r_{3}\right)=\left(-2 r_{3}, r_{1} / 2,-r_{2}\right) \\
\tau\left(y_{1}, y_{2}, y_{3}\right)=\left(y_{1},-y_{3},-y_{2}\right), \sigma\left(y_{1}, y_{2}, y_{3}\right)=\left((1 / 16) y_{3},-16 y_{1},-y_{2}\right) .
\end{gathered}
$$

Thus the only $N(H)$-invariant lines in $R$ resp. $T$ are the ones spanned by $(2,1,-1)$ resp. $(-1,16,-16)$ on which $\tau$ acts by multiplication by -1 resp. by +1 and hence

$$
R=R_{(\epsilon)} \oplus R_{(\theta)}, T=T_{\left(\chi_{0}\right)} \oplus T_{(\theta)} .
$$

We see that the morphism

$$
\pi: \mathbb{P}(M)-\left\{x_{1} x_{2} x_{3}=0\right\}
$$

$\rightarrow R \times \mathbb{P}\left(T \oplus V(8)_{\left(\chi_{0}\right)} \oplus V(8)_{(\theta)} \oplus V(0)_{\left(\chi_{0}\right)} \oplus V(4)_{(\theta)}\right) \simeq R \times \mathbb{P}^{8}$,

$$
\pi(x, s):=\left(\left(\frac{x_{4}}{x_{1}}, \frac{x_{5}}{x_{2}}, \frac{x_{6}}{x_{3}}\right),\left(\frac{x_{2} x_{3}}{x_{1}}: \frac{x_{3} x_{1}}{x_{2}}: \frac{x_{1} x_{2}}{x_{3}}\right)\right.
$$

$$
\left.: x_{7}: x_{8}: x_{9}: s_{0}: s_{1}: s_{2}\right)
$$

is $N(H)$-equivariant, dominant, and all fibres are $H$-orbits. If we consider $\left(x_{7}, x_{8}, x_{9}, s_{0}, s_{1}, s_{2}\right)$ as coordinates in $V(8)_{\left(\chi_{0}\right)} \oplus V(8)_{(\theta)} \oplus$ $V(0)_{\left(\chi_{0}\right)} \oplus V(4)_{(\theta)}$ in the target of the map $\pi$ (as we do in formula (32)) we denote them by $\left(y_{7}, y_{8}, y_{9}, y_{10}, y_{11}, y_{12}\right)$ to achieve consistency with Kat2.

How do we get equations which define the image

$$
\pi\left(\mathbb{P} \tilde{\mathcal{Q}}_{\lambda} \cap\left\{x_{1} x_{2} x_{3} \neq 0\right\}\right) \subset R \times\left(\mathbb{P}^{8}-\left\{y_{1} y_{2} y_{3}=0\right\}\right)
$$

in $\mathbb{P}^{8}-\left\{y_{1} y_{2} y_{3}=0\right\}$ from the quadrics $Q_{1}(x, s), \ldots, Q_{5}(x, s)$ in formula (26)? We can set $s_{3}=s_{4}=s_{5}=0$ in $Q_{1}, \ldots, Q_{5}$ to obtain equations $\bar{Q}_{1}, \ldots, \bar{Q}_{5}$ for $\mathbb{P} \tilde{\mathcal{Q}}_{\lambda}$ in $\mathbb{P}(M)$; the point is now that the quantities

$$
\bar{Q}_{1}, \bar{Q}_{2}, \frac{\bar{Q}_{3}}{x_{1}}, \frac{\bar{Q}_{4}}{x_{2}}, \frac{\bar{Q}_{4}}{x_{3}}
$$


are $H$-invariant (as one sees from the equations in Appendix B). Moreover, the map

$$
\pi: \mathbb{P}(M)-\left\{x_{1} x_{2} x_{3}=0\right\} \rightarrow R \times\left(\mathbb{P}^{8}-\left\{y_{1} y_{2} y_{3}=0\right\}\right)
$$

is a geometric quotient for the action of $H$ on the source (by $[\mathrm{Po}-\mathrm{Vi}]$, Thm. 4.2), so we can write

$$
\begin{gathered}
\bar{Q}_{1}=q_{1}\left(r_{1}, \ldots, y_{12}\right), \bar{Q}_{2}=q_{2}\left(r_{1}, \ldots, y_{12}\right), \frac{\bar{Q}_{3}}{x_{1}}=q_{3}\left(r_{1}, \ldots, y_{12}\right), \\
\frac{\bar{Q}_{4}}{x_{2}}=q_{4}\left(r_{1}, \ldots, y_{12}\right), \frac{\bar{Q}_{4}}{x_{3}}=q_{5}\left(r_{1}, \ldots, y_{12}\right)
\end{gathered}
$$

where $q_{1}, \ldots, q_{5}$ are polynomials in $\left(r_{1}, r_{2}, r_{3}\right),\left(y_{1}, y_{2}, y_{3}, y_{7}, \ldots, y_{12}\right)$ which one may find written out in Appendix B. Here we just want to emphasize their structural properties which will be most important for the subsequent arguments:

(1) The polynomials $q_{1}, q_{2}$ are homogeneous of degree 2 in the set of variables $\left(y_{1}, \ldots, y_{12}\right)$; the coefficients of the monomials in the $y$ 's are (inhomogeneous) polynomials of degrees $\leq 2$ in $r_{1}, r_{2}, r_{3}$. For $r_{1}=r_{2}=r_{3}=0, q_{1}, q_{2}$ do not vanish identically.

(2) The polynomials $q_{3}, q_{4}, q_{5}$ are homogeneous linear in $\left(y_{1}, \ldots, y_{12}\right)$; the coefficients of the monomials in the $y$ 's are (inhomogeneous) polynomials of degrees $\leq 2$ in $r_{1}, r_{2}, r_{3}$. For $r_{1}=r_{2}=r_{3}=0$, $q_{3}, q_{4}, q_{5}$ do not vanish identically.

Theorem 3.2.1. Let $\tilde{Y}_{\lambda}$ be the subvariety of $R \times \mathbb{P}^{8}$ defined by the equations $q_{1}=q_{2}=q_{3}=q_{4}=q_{5}=0$. There is an irreducible $N(H)$ invariant component $Y_{\lambda}$ of $\tilde{Y}_{\lambda}$ with $\pi\left(\left[x^{0}\right]\right) \in Y_{\lambda}$, where $x^{0}:=13 i\left(5 e_{7}+\right.$ $\left.e_{9}\right)+5\left(4 e_{1}-i e_{2}+e_{3}\right)$, such that

$$
\mathbb{C}\left(\mathbb{P} \mathcal{Q}_{\lambda}\right)^{N(H)} \simeq \mathbb{C}\left(Y_{\lambda}\right)^{N(H)} .
$$

Proof. The variety $Y_{\lambda}$ will be the closure of the image $\pi\left(\mathbb{P} \mathcal{Q}_{\lambda} \cap\left\{x_{1} x_{2} x_{3} \neq\right.\right.$ $0\})$ in $R \times \mathbb{P}^{8}$.

It remains to see that $x^{0} \in \mathcal{Q}_{\lambda}$. Recall from Theorem 3.1.5 that $\mathcal{Q}_{\lambda}$ is the unique irreducible component of $\tilde{\mathcal{Q}}_{\lambda}$ passing through the $N(H)$ invariant point $5 e_{7}+e_{9}$, and that this point is a nonsingular point on $\tilde{\mathcal{Q}}_{\lambda}$; thus, if we can find an irreducible subvariety of $\tilde{\mathcal{Q}}_{\lambda}$ which contains both $5 e_{7}+e_{9}$ and $x^{0}$, we are done. The sought-for subvariety is $\tilde{\mathcal{Q}}_{\lambda} \cap V(8)^{\sigma}$, where $V(8)^{\sigma}$ are the elements in $V(8)$ invariant under 
$\sigma \in N(H)$. One sees that $x^{0}$ and $5 e_{7}+e_{9}$ lie on it, and computing

$$
\begin{gathered}
V(8)^{\sigma}=\left\langle 5 e_{7}+e_{9}, 8 e_{4}-i e_{5}-e_{6}, 4 e_{1}-i e_{2}+e_{3}\right\rangle, \\
V(4)^{\sigma}=\left\langle 2\left(z_{1}^{4}-z_{2}^{4}\right)+4\left(z_{1}^{3} z_{2}+z_{1} z_{2}^{3}\right)+4 i\left(z_{1}^{3} z_{2}-z_{1} z_{2}^{3}\right)\right\rangle,
\end{gathered}
$$

and using $\delta_{\lambda}\left(V(8)^{\sigma}\right) \subset V(4)^{\sigma}$, we find that $\tilde{\mathcal{Q}}_{\lambda} \cap V(8)^{\sigma}$ is a quadric in $V(8)^{\sigma}$ which is easily checked to be irreducible.

Thus it remains to prove the rationality of $Y_{\lambda} / N(H)=Y_{\lambda} / \mathfrak{S}_{3}$.

3.3. Inner projections and the "no-name" method. The variety $\tilde{Y}_{\lambda}$ comes with the two projections

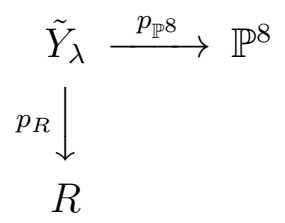

Recall from (32) that $N:=\mathbb{P}\left(V(8)_{\theta} \oplus V(4)_{\theta}\right) \subset \mathbb{P}^{8}$ is an $N(H)$-invariant 3-dimensional projective subspace of $\mathbb{P}^{8}$. We will show $\mathbb{C}\left(Y_{\lambda}\right)^{N(H)} \simeq$ $\mathbb{C}(R \times N)^{N(H)}$ via the following theorem.

Theorem 3.3.1. There is an open $N(H)$-invariant subset $R_{0} \subset R$ containing $0 \in R$ with the following properties:

(1) For all $r \in R_{0}$ the fibre $p_{R}^{-1}(r) \subset \tilde{Y}_{\lambda}$ is irreducible of dimension 3 , and $p_{R}^{-1}\left(R_{0}\right)$ is an open $N(H)$-invariant subset of $Y_{\lambda}$.

(2) There exist $N(H)$-sections $\sigma_{1}, \sigma_{2}$ of the $N(H)$-equivariant projection $R_{0} \times \mathbb{P}^{8} \rightarrow R_{0}$ such that $N(r):=\left\langle\sigma_{1}(r), \sigma_{2}(r),(1: 0:\right.$ $0: \cdots: 0),(0: 1: 0: \cdots: 0),(0: 0: 1: 0: \cdots: 0)\rangle \subset \mathbb{P}^{8}$, $r \in R_{0}$, is an $N(H)$-invariant family of 4-dimensional projective subspaces in $\mathbb{P}^{8}$ with the properties:

(i) $N(r)$ is disjoint from $N$ for all $r \in R_{0}$.

(ii) The fibre $p_{\mathbb{P}^{8}}\left(p_{R}^{-1}(r)\right) \subset \mathbb{P}^{8}$ contains the line $\left\langle\sigma_{1}(r), \sigma_{2}(r)\right\rangle \subset$ $N(r)$ for all $r \in R_{0}$.

(iii) The projection $\pi_{r}: \mathbb{P}^{8} \rightarrow N$ from $N(r)$ to $N$ maps the fibre $p_{\mathbb{P}^{8}}\left(p_{R}^{-1}(r)\right) \subset \mathbb{P}^{8}$ dominantly onto $N$ for all $r \in R_{0}$.

Before turning to the proof, let us note the following corollary.

Corollary 3.3.2. One has the field isomorphism

$$
\mathbb{C}\left(Y_{\lambda}\right)^{N(H)} \simeq \mathbb{C}(R \times N)^{N(H)},
$$

and the latter field is rational. Hence $\mathfrak{M}_{3}$ is rational.

Proof. (of corollary) The $N(H)$-invariant set $p_{R}^{-1}\left(R_{0}\right)$ is an open subset of $Y_{\lambda}$. Let us see that the projection $\pi_{r}: F_{r}:=p_{\mathbb{P} 8}\left(p_{R}^{-1}(r)\right) \rightarrow N$ is 
birational. In fact, $F_{r}$ is of dimension 3 and irreducible and the intersection of a 3-codimensional linear subspace and two quadrics in $\mathbb{P}^{8}$. Moreover, $F_{r} \cap N(r)$ contains a line $L_{r}$ by Theorem 3.3.1 (2), (ii). Thus for a general point $P$ in $N, F_{r} \cap\left\langle L_{r}, P\right\rangle$ consists of $L_{r}$ and a single point (namely the point of intersection of the two lines which are the residual intersections of each of the two quadrics defining $F_{r}$ with $\left\langle L_{r}, P\right\rangle$, the other component being $L_{r}$ itself). Thus $\pi_{r}$ is generically one-to-one whence birational.

Thus one has a birational $N(H)$-isomorphism $p_{R}^{-1}\left(R_{0}\right) \rightarrow R_{0} \times N$, given by sending $(r,[y])$ to $\left(r, \pi_{r}([y])\right)$. Thus one gets the field isomorphism in Corollary 3.3.2.

By the no-name lemma (cf. e.g. Dol1], section 4), $\mathbb{C}(R \times N)^{N(H)} \simeq$ $\mathbb{C}(N)^{N(H)}\left(T_{1}, T_{2}, T_{3}\right)$, where $T_{1}, T_{2}, T_{3}$ are indeterminates, thus it suffices to show that the quotient of $N$ by $N(H)$ is stably rational of level $\leq 3$. This in turn follows from the same lemma, since clearly, if we take the representation of $\mathfrak{S}_{3}$ in $\mathbb{C}^{3}$ by permutation of coordinates, the quotient of $\mathbb{P}\left(\mathbb{C}^{3}\right)$ by $\mathfrak{S}_{3}$, a unirational surface, is rational.

Proof. (of theorem) The proof will be given in several steps.

Step 1. (Irreducibility of the fibre over 0) We have to show that the variety $\left.p_{\mathbb{P}^{8}} p_{R}^{-1}(0)\right) \subset \mathbb{P}^{8}$ is irreducible and 3-dimensional. We have explicit equations for it (namely the ones that arise if we substitute $r_{1}=r_{2}=r_{3}=0$ in $q_{1}, \ldots, q_{5}$, which are thus 3 linear and 2 quadratic equations); the assertions can then be checked with a computer algebra system such as Macaulay 2. Recall from Theorem 3.2.1 that $Y_{\lambda}$ contains $\pi\left(\left[x^{0}\right]\right)$. In fact,

$$
\pi\left(\left[x^{0}\right]\right)=\left((0,0,0),\left(-\frac{5}{4}: 20:-20: 65: 0: 13: 0: 0: 0\right)\right)
$$

as follows from the definition of $x^{0}$ in Theorem 3.2.1 and the definition of $\pi$ in (32). Thus $\pi\left(\left[x^{0}\right]\right)$ lies in the fibre over 0 of $p_{R}^{-1}$ and thus, since there is an open subset around 0 in $R$ over which the fibres are irreducible and 3-dimensional, assertion (1) of Theorem 3.3.1 is established.

Step 2. (Construction of $\sigma_{1}$ ) To obtain $\sigma_{1}$, we just assign to $r \in R$ the point $\left(r, \sigma_{1}(r)\right)$ with $\sigma_{1}(r)=(0: 0: 0: 0: 0: 0: 1: 0: 0)$, i.e. $y_{10}=1$, the other $y$ 's being 0 . This always is in the fibre $p_{\mathbb{P}^{8}}\left(p_{R}^{-1}(r)\right)$ as one sees on substituting in the equations $q_{1}, \ldots, q_{5}$. Moreover, this is an $N(H)$-section, since $y_{10}$ is a coordinate in the space $V(0)_{\chi_{0}}$ in formula (32).

Step 3. (Construction of $\sigma_{2}$; decomposition of $V:=\mathbb{P}\left(\delta_{\lambda}^{-1}(0) \cap V(8)\right.$ ) ) The construction of a section $\sigma_{2}, \sigma_{2}(r)=\left(\sigma_{2}^{(1)}(r): \cdots: \sigma_{2}^{(9)}(r)\right)$, 
involves a little more work. Let us look back at the construction of $Y_{\lambda}$ in subsection 3.2 for this, especially the definition of the projection $\pi$ in formula (32), and the decomposition of the linear subspace $M \subset V(8) \oplus V(0) \oplus V(4)$. By definition of $R$, the family of codimension 3 linear subspaces

$$
L(r):=\left\{[(x, s)] \mid x_{4}=r_{1} x_{1}, x_{5}=r_{2} x_{2}, x_{6}=r_{3} x_{3}\right\} \subset \mathbb{P}(M),
$$

$r=\left(r_{1}, r_{2}, r_{3}\right) \in R$, is $N(H)$-invariant, i.e. $g L(r)=L(g r)$, for $g \in$ $N(H)$. It is natural to intersect this family with $\mathbb{P}\left(\delta_{\lambda}^{-1}(0) \cap V(8)\right)$ which, as we will see, has dimension 3 and look for an $H$-orbit $\mathfrak{O}_{r}$ in the intersection of $\mathbb{P}\left(\delta_{\lambda}^{-1}(0) \cap V(8)\right)$ with the open set of $L(r)$ where $x_{1} x_{2} x_{3} \neq 0$. Moreover, we will see that for $r=0$, the point $\left[x^{0}\right]$ is in this intersection. Thus passing to the quotient we may put

$$
\left(r, \sigma_{2}(r)\right):=\pi\left(\mathfrak{O}_{r}\right)
$$

to obtain a $\sigma_{2}$ with the required properties. Indeed, note that we will have $\sigma_{2}^{(7)}(r)=\sigma_{2}^{(8)}=\sigma_{2}^{(9)}=0$ which ensures that $\sigma_{2}$ and $\sigma_{1}$ span a line. Moreover,

$$
\sigma_{2}(0)=\left(-\frac{5}{4}: 20:-20: 65: 0: 13: 0: 0: 0\right)
$$

by formula (34), which allows us to check assertions (2), (i) and (iii) of Theorem 3.3.1, which are open properties on the base $R$, by explicit computation for the fibre over 0. Property (2), (ii) stated in the theorem is clear by construction. Let us now carry out this program. We will start by explicitly decomposing $V:=\mathbb{P}\left(\delta_{\lambda}^{-1}(0) \cap V(8)\right)$ into irreducible components.

To guess what $V$ might be, note that according to the definition of $\delta_{\lambda}$ in Lemma 2.4.4, $\delta_{\lambda}$ vanishes on $f_{8} \in V(8)$ if for the transvectant $\psi_{6}$ one has $\psi_{6}\left(f_{8}, f_{8}\right)=0$; but looking back at the definition of transvectants in formula (20), we see that $\psi_{6}: V(8) \times V(8) \rightarrow V(4)$ vanishes if $f_{8}$ is a linear combination of $z_{1}^{8}, z_{1}^{7} z_{2}$ and $z_{1}^{6} z_{2}^{2}$ (since we differentiate at least 3 times with respect to $z_{2}$ in one factor in the summands in formula (20)). Thus $X_{1}:=\overline{\mathrm{PSL}_{2} \mathbb{C} \cdot\left\langle z_{1}^{8}, z_{1}^{7} z_{2}, z_{1}^{6} z_{2}^{2}\right\rangle}$, the variety of forms of degree 8 with a six-fold zero, is contained in $V$, and one computes that the differential of $\left.\delta_{\lambda}\right|_{V(8)}$ in $z_{1}^{6} z_{2}^{2}$ is surjective, so that $X_{1}$ is an irreducible component of $V$.

The dimension of $X_{1}$ is clearly three. Weyman, in [Wey, Cor. 4, computed the Hilbert function of $X_{p, g}$, the variety of binary forms of degree $g$ having a root of multiplicity $\geq p$ which is

$$
H\left(X_{p, g}, d\right)=(d p+1)\left(\begin{array}{c}
g-p+d \\
g-p
\end{array}\right)-(d(p+1)-1)\left(\begin{array}{c}
g-p+d-1 \\
g-p-1
\end{array}\right) .
$$


For $d=6, g=8$, the leading term in $d$ in this expression is $3 d^{3}$, which shows

$$
\operatorname{deg} X_{1}=18 .
$$

Moreover, we know already that $5 e_{7}+e_{9}$ is in $V$ from the proof of Theorem 3.1.5; thus set $X_{2}:=\overline{\mathrm{PSL}_{2} \mathbb{C} \cdot\left\langle 5 e_{7}+e_{9}\right\rangle}$. We know that the stabilizer of $5 e_{7}+e_{9}$ in $\mathrm{PSL}_{2} \mathbb{C}$ contains $N(H)$ because $5 e_{7}+e_{9}=$ $5 z_{1}^{8}+5 z_{2}^{8}+70 z_{1}^{4} z_{2}^{4}$ spans the $N(H)$-invariants in $V(8)$ by Lemma 3.1.4. The claim is that the stabilizer is not larger. An easy way to check this is to use the beautiful theory developed in [Ol], p. $188 \mathrm{ff}$., using differential invariants and signature curves, which allows the explicit determination of the order of the symmetry group of a complex binary form. More precisely we have (cf. [Ol], Cor. 8.68):

Theorem 3.3.3. Let $Q(p)$ be a binary form of degree $n$ (written in terms of the inhomogeneous coordinate $p=z_{1} / z_{2}$ ) which is not equivalent to a monomial. Then the cardinality $k$ of the symmetry group of $Q(p)$ satisfies

$$
k \leq 4 n-8,
$$

provided that $U$ is not a constant multiple of $H^{2}$, where $U$ and $H$ are the following polynomials in $p: H:=(1 / 2)(Q, Q)^{(2)}, T:=(Q, H)^{(1)}$, $U:=(Q, T)^{(1)}$ where, if $Q_{1}$ is a binary form of degree $n_{1}$, and $Q_{2}$ is a binary form of degree $n_{2}$, we put

$$
\begin{gathered}
\left(Q_{1}, Q_{2}\right)^{(1)}:=n_{2} Q_{1}^{\prime} Q_{2}-n_{1} Q_{1} Q_{2}^{\prime}, \\
\left(Q_{1}, Q_{2}\right)^{(2)}:=n_{2}\left(n_{2}-1\right) Q_{1}^{\prime \prime} Q_{2}-2\left(n_{2}-1\right)\left(n_{1}-1\right) Q_{1}^{\prime} Q_{2}^{\prime} \\
+n_{1}\left(n_{1}-1\right) Q_{1} Q_{2}^{\prime \prime} .
\end{gathered}
$$

(these are certain transvectants).

Applying this result in our case, we find the upper bound 24 for the symmetry group of $5 e_{7}+e_{9}$, which is indeed the order of $N(H)=\mathfrak{S}_{4}$. $X_{2}$ is irreducible of dimension 3 , and computing that the differential of $\left.\delta_{\lambda}\right|_{V(8)}$ is surjective in $5 e_{7}+e_{9}$, we get that $X_{2}$ is another irreducible component of $V$. But let us intersect $X_{2}$ with the codimension 3 linear subspace in $V(8)$ consisting of forms with zeroes $\zeta_{1}, \zeta_{2}, \zeta_{3} \in \mathbb{P}^{1}$; there is a unique projectivity carrying these to three roots of $5 e_{7}+e_{9}$, which are all distinct, thus there are $8 \cdot 7 \cdot 6$ such projectivities, and $\operatorname{deg} X_{2} \geq$ $(8 \cdot 7 \cdot 6) /|N(H)|$. But one checks easily that $V$ itself has dimension 3 and is the intersection of 5 quadrics in $\mathbb{P}(V(8))$, thus has degree $\leq 32$. Thus we must have

$$
\operatorname{deg} X_{2}=14, V=X_{1} \cup X_{2}, \operatorname{deg} V=32 .
$$


Note also that

$$
\left[x^{0}\right] \in X_{2} \cap L(0) .
$$

In fact, from the proof of Theorem 3.2.1, we know $\left[x^{0}\right] \in V$, and $\left[x^{0}\right] \in L(0)$ being clear, we just check that $x^{0}$ has no root of multiplicity $\geq 6$.

Step 4. (Construction of $\sigma_{2}$; intersecting $V$ with a family of linear spaces in $\mathbb{P}(M))$ Let $L^{0}(r)$ be the open subset of $L(r) \subset \mathbb{P}(M)$ where $x_{1} x_{2} x_{3} \neq 0$. According to the strategy outlined at the beginning of Step 3, we would like to compute the cardinalities

$$
\left|L^{0}(r) \cap X_{1}\right|, \quad\left|L^{0}(r) \cap X_{2}\right|
$$

for $r$ varying in a small neighbourhood of 0 in $R$. It is, however, easier from a computational point of view to determine the number of intersection points of $X_{1}$ resp. $X_{2}$ with certain boundary components of $L^{0}(r)$ in $L(r)$ first; the preceding cardinalities will afterwards fall out as the residual quantities needed to have $\operatorname{deg} X_{1}=18, \operatorname{deg} X_{2}=14$. Thus let us introduce the following additional strata of $L(r) \backslash L^{0}(r)$ :

$$
\begin{gathered}
L_{0}:=\left\{[(x, s)] \mid x_{1}=x_{2}=x_{3}=x_{4}=x_{5}=x_{6}=0\right\}, \\
L_{1}(r):=\left\{[(x, s)] \mid x_{1} \neq 0, x_{4}=r_{1} x_{1}, x_{2}=x_{3}=x_{5}=x_{6}=0\right\}, \\
L_{2}(r):=\left\{[(x, s)] \mid x_{2} \neq 0, x_{5}=r_{2} x_{2}, x_{1}=x_{3}=x_{4}=x_{6}=0\right\}, \\
L_{3}(r):=\left\{[(x, s)] \mid x_{3} \neq 0, x_{6}=r_{3} x_{3}, x_{1}=x_{2}=x_{4}=x_{5}=0\right\}, \\
\tilde{L}_{1}(r):=\left\{[(x, s)] \mid x_{2} x_{3} \neq 0, x_{5}=r_{2} x_{2}, x_{6}=r_{3} x_{3}, x_{1}=x_{4}=0\right\} \\
\tilde{L}_{2}(r):=\left\{[(x, s)] \mid x_{1} x_{3} \neq 0, x_{4}=r_{1} x_{1}, x_{6}=r_{3} x_{3}, x_{2}=x_{5}=0\right\} \\
\tilde{L}_{3}(r):=\left\{[(x, s)] \mid x_{1} x_{2} \neq 0, x_{4}=r_{1} x_{1}, x_{5}=r_{2} x_{2}, x_{3}=x_{6}=0\right\} .
\end{gathered}
$$

$L(r)$ is the disjoint union of these and $L^{0}(r)$. From the equations describing $\delta_{\lambda}$ one sees that $V$ is defined in $\mathbb{P}(V(8))$ with coordinates 
$x_{1}, \ldots, x_{9}$ by

$$
\begin{gathered}
-192 x_{6}^{2}-192 x_{3} x_{6}+384 x_{3}^{2}-192 x_{5}^{2}-192 x_{2} x_{5}+384 x_{2}^{2} \\
-12 x_{1} x_{4}+12 x_{7} x_{8}+180 x_{8} x_{9}=0 \\
64 x_{6}^{2}-192 x_{3} x_{6}-128 x_{3}^{2}-64 x_{5}^{2}+192 x_{2} x_{5}+128 x_{2}^{2} \\
-2 x_{4}^{2}+16 x_{1}^{2}+2 x_{7}^{2}-16 x_{8}^{2}-50 x_{9}^{2}=0 \\
96 x_{5} x_{6}-672 x_{3} x_{5}-672 x_{2} x_{6}+1248 x_{2} x_{3} \\
-12 x_{1} x_{7}+12 x_{4} x_{8}+180 x_{1} x_{9}=0 \\
6 x_{4} x_{6}+42 x_{3} x_{4}+84 x_{1} x_{6}+156 x_{1} x_{3} \\
-6 x_{5} x_{7}-42 x_{2} x_{7}+24 x_{5} x_{8}-264 x_{2} x_{8}+30 x_{5} x_{9}-30 x_{2} x_{9}=0 \\
-6 x_{4} x_{5}-42 x_{2} x_{4}+84 x_{1} x_{5}+156 x_{1} x_{2} \\
+6 x_{6} x_{7}+42 x_{3} x_{7}+24 x_{6} x_{8}-264 x_{3} x_{8}-20 x_{6} x_{9}+30 x_{3} x_{9}=0
\end{gathered}
$$

and thus

$$
\tilde{L}_{i}(r) \cap V=\emptyset \quad \forall i=1,2,3
$$

for $r$ in a Zariski open neighbourhood of $0 \in R$ (for $\tilde{L}_{1}(r)$ consider equation (44) and assume $96 r_{2} r_{3}-672 r_{2}-672 r_{3}+1248 \neq 0$, for $\tilde{L}_{2}(r)$ we see that (45) cannot hold if $6 r_{1} r_{3}+42 r_{1}+84 r_{3}+156 \neq 0$, and for $\tilde{L}_{3}(r)$ equation (46) is impossible provided that $-6 r_{1} r_{2}-42 r_{1}+84 r_{2}+156 \neq$ $0)$.

Let us consider the intersection $V \cap L_{0}$. We have to solve the equations

$$
12 x_{7} x_{8}+180 x_{8} x_{9}=0, \quad 2 x_{7}^{2}-16 x_{8}^{2}-50 x_{9}^{2}=0,
$$

which have the four distinct solutions $\left(x_{7}, x_{8}, x_{9}\right)=(5,0, \pm 1),\left(x_{7}, x_{8}, x_{9}\right)=$ $(15, \pm 5,-1)$, whence

$$
L_{0} \cap V=\left\{\left[5 e_{7} \pm e_{9}\right],\left[15 e_{7} \pm 5 e_{8}-e_{9}\right]\right\} .
$$

We will also have to determine the intersection $V \cap L_{1}(r)$ explicitly. We have to solve the equations

$$
\begin{gathered}
-12 r_{1} x_{1}^{2}+12 x_{7} x_{8}+180 x_{8} x_{9}=0, \\
-2 r_{1}^{2} x_{1}^{2}+16 x_{1}^{2}+2 x_{7}^{2}-16 x_{8}^{2}-50 x_{9}^{2}=0, \\
-12 x_{1} x_{7}+12 r_{1} x_{1} x_{8}+180 x_{1} x_{9}=0,
\end{gathered}
$$

in the variables $x_{1}, x_{7}, x_{8}, x_{9}$. We can check (e.g. with Macaulay 2) that the subscheme they define has dimension 0 (and degree 8) for $r_{1}=0$. We already know four solutions with $x_{1}=0$, namely the ones given in formula (48). Then it suffices to check that

$\left(x_{1}, x_{7}, x_{8}, x_{9}\right)=\left( \pm 1, r_{1}, 1,0\right),\left(x_{1}, x_{7}, x_{8}, x_{9}\right)=\left( \pm a,\left(90-5 r_{1}^{2}\right),-5 r_{1}, 6\right)$, 
where $a$ is a square-root of $25\left(r_{1}^{2}-36\right.$ ), are also solutions (with $x_{1} \neq 0$ in a neighbourhood of 0 in $R$, and obviously all distinct there). Thus

$$
\begin{gathered}
L_{1}(r) \cap V=\left\{\left[ \pm\left(e_{1}+r_{1} e_{4}\right)+r_{1} e_{7}+e_{8}\right],\right. \\
\left.\left[ \pm\left(a e_{1}+r_{1} a e_{4}\right)+\left(90-5 r_{1}^{2}\right) e_{7}-5 r_{1} e_{8}+6 e_{9}\right]\right\} .
\end{gathered}
$$

We still have to see how the intersection points $L_{0} \cap V$ and $L_{1}(r) \cap V$ are distributed among $X_{1}$ and $X_{2}$ : Suppose $f \in V(8)$ is a binary octic such that $[f] \in L_{0} \cap \mathbb{P}(V(8))$ or $[f] \in L_{1}(r) \cap \mathbb{P}(V(8))$; then $f$ is a linear combination of the binary octics $e_{1}, e_{4}, e_{7}, e_{8}, e_{9}$ defined in (22), which involve only even powers of $z_{1}$ and $z_{2}$; thus if $(a: b) \in \mathbb{P}^{1}$ is a root of one of them, so is its negative $(a:-b)$ whence

$[f]$ lies in $X_{1}$ if and only if $(1: 0)$ or $(0: 1)$ is a root of multiplicity $\geq 6$.

Applying this criterion, we get, using (48) and (49)

$$
\begin{gathered}
L_{0} \cap X_{1}=\emptyset, L_{0} \cap X_{2}=\left\{\left[5 e_{7} \pm e_{9}\right],\left[15 e_{7} \pm 5 e_{8}-e_{9}\right]\right\}, \\
L_{1}(r) \cap X_{1}=\left\{\left[ \pm\left(e_{1}+r_{1} e_{4}\right)+r_{1} e_{7}+e_{8}\right]\right\}, \\
L_{1}(r) \cap X_{2}=\left\{\left[ \pm\left(a e_{1}+r_{1} a e_{4}\right)+\left(90-5 r_{1}^{2}\right) e_{7}-5 r_{1} e_{8}+6 e_{9}\right]\right\} .
\end{gathered}
$$

The reader may be glad to hear now that we do not have to repeat this entire procedure for $L_{2}(r)$ and $L_{3}(r)$; in fact, $L_{1}(r), L_{2}(r), L_{3}(r)$ are permuted by $N(H)$ in the following way: For the element $\sigma \in N(H)$ we have

$$
\sigma \cdot L_{1}(r)=L_{2}(\sigma \cdot r), \quad \sigma \cdot L_{2}(r)=L_{3}(\sigma \cdot r), \quad \sigma \cdot L_{3}(r)=L_{1}(\sigma \cdot r),
$$

which follows from (30) (and (28)) and the definition of $R$. Thus we get that generally for $i=1,2,3$

$$
L_{i}(r) \cap X_{1}=\left\{P_{1}(r), P_{2}(r)\right\}, L_{i}(r) \cap X_{2}=\left\{Q_{1}(r), Q_{2}(r)\right\}
$$

where $P_{1}(r), P_{2}(r), Q_{1}(r), Q_{2}(r)$ are mutually distinct points, and this is valid in a Zariski open $N(H)$-invariant neighbourhood of $0 \in R$. It remains to check that

$$
L(0) \cap V \text { consists of } 32 \text { reduced points. }
$$

We check (with Macaulay 2) that if we substitute $x_{4}=x_{5}=x_{6}=0$ in equations (42)-(46), they define a zero-dimensional reduced subscheme of degree 32 in the projective space with coordinates $x_{1}, x_{2}, x_{3}, x_{7}, x_{8}, x_{9}$. Taking into account $(47),(50),(51)$, we see that all the intersections in equations (50), (51) are free of multiplicities in an open $N(H)$-invariant neighbourhood of $0 \in R$ and moreover, since $\operatorname{deg} X_{1}=18, \operatorname{deg} X_{2}=14$, we must have there

$L^{0}(r) \cap X_{1}$ consists of 12 reduced points, and $L^{0}(r) \cap X_{2}$ consists of 4 reduced points. 
Now these 4 points make up the $H$-orbit $\mathfrak{O}_{r}$ we wanted to find in Step 3: Clearly $L^{0}(r) \cap X_{2}$ is $H$-invariant, and $H$ acts with trivial stabilizers in $L^{0}(r)$ (as is clear from $(29)$ ). Thus we have completed the program outlined at the beginning of Step 3. It just remains to notice that $\left[x^{0}\right] \in X_{2} \cap L^{0}(0)$. This is clear since $\left[x^{0}\right] \in V$, but $x^{0}$ does not have a root of multiplicity $\geq 6$.

Step 5. (Verification of the properties of $N(r)$ ) For the completion of the proof of Theorem 3.3.1, it remains to verify the properties of the subspace $N(r)$ in parts (2), (i) and (iii) of that theorem. First of all, it is clear that

$$
\begin{gathered}
N(r)=\left\langle\sigma_{1}(r), \sigma_{2}(r),(1: 0: 0: \cdots: 0),\right. \\
(0: 1: 0: \cdots: 0),(0: 0: 1: \cdots: 0)\rangle
\end{gathered}
$$

is $N(H)$-invariant in the sense that $g \cdot N(r)=N(g \cdot r)$ for $g \in N(H)$ by the construction of $\sigma_{1}, \sigma_{2}$ and because the last three vectors in the preceding formula are a basis in the invariant subspace $\mathbb{P}(T) \subset \mathbb{P}^{8}$ (where by (31) $\left.T=T_{\left(\chi_{0}\right)} \oplus T_{(\theta)}\right)$. Moreover, by the definition of $\sigma_{1}$ in Step 2, and the formula (37) for $\sigma_{2}(0)$, one has $\operatorname{dim} N(0)=4$, which thus holds also for $r \in R$ sufficiently close to 0 .

Recall that $N$ was defined to be $N:=\mathbb{P}\left(V(8)_{(\theta)} \oplus V(4)_{(\theta)}\right) \subset \mathbb{P}^{8}$, and as such can be described in terms of the coordinates $\left(y_{1}: y_{2}: y_{3}: y_{7}\right.$ : $\left.y_{8}: \cdots: y_{12}\right)$ in $\mathbb{P}^{8}$ as

$$
N=\left\{y_{1}=y_{2}=y_{3}=y_{7}+7 y_{9}=y_{10}=0\right\}
$$

(cf. (24)). Thus we get that $N(0) \cap N=\emptyset$, and the same holds in an open $N(H)$-invariant neighbourhood of 0 in $R$.

For Theorem 3.3.1, (2), (iii), it suffices to check that $\pi_{0}$ maps the fibre $p_{\mathbb{P}^{8}}\left(p_{R}^{-1}(0)\right)$ dominantly onto $N$, which can be done by direct calculation. This concludes the proof.

\section{Appendix A. Collection of Formulas for SeCtion 2}

We start with some remarks on how to calculate equivariant projections, and then we give explicit formulas for the equivariant maps in section 2 .

Let $a, b$ be nonnegative integers, $m:=\min (a, b)$, and let $G:=\mathrm{SL}_{3} \mathbb{C}$. We denote the irreducible $G$-module whose highest weight has numerical labels $a, b$ by $V(a, b)$. For $k=0, \ldots, m$ we define $V^{k}:=$ $\operatorname{Sym}^{a-k} \mathbb{C}^{3} \otimes \operatorname{Sym}^{b-k}\left(\mathbb{C}^{3}\right)^{\vee}$. Let $e_{1}, e_{2}, e_{3}$ be the standard basis in $\mathbb{C}^{3}$ and $x_{1}, x_{2}, x_{3}$ the dual basis in $\left(\mathbb{C}^{3}\right)^{\vee}$.

There are $G$-equivariant linear maps $\Delta^{k}: V^{k} \rightarrow V^{k+1}$ for $k=0, \ldots, m-$ 1 and $\delta^{k}: V^{k} \rightarrow V^{k-1}$ for $k=1, \ldots, m$ given by 


$$
\Delta^{k}:=\sum_{i=1}^{3} \frac{\partial}{\partial e_{i}} \otimes \frac{\partial}{\partial x_{i}}, \quad \delta^{k}:=\sum_{i=1}^{3} e_{i} \otimes x_{i} .
$$

(The superscript $k$ thus only serves as a means to remember the sources and targets of the respective maps). If for some positive integers $\alpha, \beta$ the $G$-module $V^{k}$ contains a $G$-submodule isomorphic to $V(\alpha, \beta)$ we will denote it by $V^{k}(\alpha, \beta)$ to indicate the ambient module (this is unambiguous because it is known that all such modules occur with multiplicity one).

It is clear that $\Delta^{k}$ is surjective and $\delta^{k}$ injective; one knows that $\operatorname{ker}\left(\Delta^{k}\right)=$ $V^{k}(a-k, b-k)$ whence

$$
V^{k}=\bigoplus_{i=k}^{m} V^{k}(a-i, b-i) .
$$

We want to find a formula for the $G$-equivariant projection of $V^{0}=$ $\operatorname{Sym}^{a} \mathbb{C}^{3} \otimes \operatorname{Sym}^{b}\left(\mathbb{C}^{3}\right)^{\vee}$ onto the subspace $V^{0}(a-i, b-i)$ for $i=0, \ldots, m$. We call this linear map $\pi_{a, b}^{i}$.

We remark that, by (53), one can decompose each vector $v \in V^{0}$ as $v=v_{0}+\cdots+v_{m}$ where $v_{i} \in V^{0}(a-i, b-i)$, and this decomposition is unique. Note that

$$
\delta^{1} \ldots \delta^{i}\left(\operatorname{ker} \Delta^{i}\right)=V^{0}(a-i, b-i)
$$

so that

$$
\begin{gathered}
V^{0}=\operatorname{ker} \Delta^{0} \oplus \delta^{1}\left(\operatorname{ker} \Delta^{1}\right) \oplus \delta^{1} \delta^{2}\left(\operatorname{ker} \Delta^{2}\right) \oplus \cdots \oplus \delta^{1} \ldots \delta^{i}\left(\operatorname{ker} \Delta^{i}\right) \\
\oplus \cdots \oplus \delta^{1} \ldots \delta^{m}\left(V^{m}\right) .
\end{gathered}
$$

Of course, $\pi_{a, b}^{i}(v)=v_{i}$. It will be convenient to put

$$
L^{i}:=\delta^{1} \circ \delta^{2} \circ \cdots \circ \delta^{i} \circ \Delta^{i-1} \circ \cdots \circ \Delta^{1} \circ \Delta^{0}, \quad i=0, \ldots, m
$$

(whence $L^{0}$ is the identity) and

$$
U^{i}:=\Delta^{i-1} \circ \Delta^{i-2} \circ \cdots \circ \Delta^{0} \circ \delta^{1} \circ \cdots \circ \delta^{i-1} \circ \delta^{i}, \quad i=0, \ldots, m
$$

( $U^{0}$ being again the identity). By Schur's lemma, we have

$$
\left.U^{i}\right|_{V^{i}(a-i, b-i)}=c_{i} \cdot \operatorname{id}_{V^{i}(a-i, b-i)}
$$


for some nonzero rational number $c_{i} \in \mathbb{Q}^{*}$. This is easy to calculate: For example, since $e_{1}^{a-i} \otimes x_{2}^{b-i} \in \operatorname{ker} \Delta^{i}=V^{i}(a-i, b-i)$, we have that $c_{i}$ is the unique number such that

$$
U^{i}\left(e_{1}^{a-i} \otimes x_{2}^{b-i}\right)=c_{i} \cdot e_{1}^{a-i} \otimes x_{2}^{b-i} .
$$

We will now calculate $\pi_{a, b}^{m-l}$ for $l=0, \ldots, m$ by induction on $l$; the case $l=0$ can be dealt with as follows:

Write $v=v_{1}+\cdots+v_{m} \in V^{0}$ as before. Then $v_{m}=\delta^{1} \delta^{2} \ldots \delta^{m}\left(u_{m}\right)$ for some $u_{m} \in V^{m}$. Now

$$
\begin{gathered}
L^{m}(v)=L^{m}\left(v_{m}\right)=L^{m}\left(\delta^{1} \delta^{2} \ldots \delta^{m}\left(u_{m}\right)\right) \\
=\delta^{1} \delta^{2} \ldots \delta^{m} \circ U^{m}\left(u_{m}\right)=c_{m} v_{m}
\end{gathered}
$$

so we set

$$
\pi_{a, b}^{m}:=\frac{1}{c_{m}} L^{m} .
$$

Now assume, by induction, that $\pi_{a, b}^{m-l}, \pi_{a, b}^{m-l+1}, \ldots, \pi_{a, b}^{m}$ have already been determined. We show how to calculate $\pi_{a, b}^{m-l-1}$.

Now, by $(54), v_{m-l-1} \in \delta^{1} \ldots \delta^{m-l-1}\left(\operatorname{ker} \Delta^{m-l-1}\right)$. We write $v_{m-l-1}=$ $\delta^{1} \ldots \delta^{m-l-1}\left(u_{m-l-1}\right)$, for some $u_{m-l-1} \in \operatorname{ker} \Delta^{m-l-1}=V^{m-l-1}(a-$ $(m-l-1), b-(m-l-1))$, and using (57) we get

$$
\begin{gathered}
L^{m-l-1}\left(v-\sum_{i=0}^{l} \pi_{a, b}^{m-i}(v)\right)=L^{m-l-1}\left(v_{0}+v_{1}+\cdots+v_{m-l-1}\right) \\
=L^{m-l-1}\left(v_{m-l-1}\right)=L^{m-l-1}\left(\delta^{1} \ldots \delta^{m-l-1}\left(u_{m-l-1}\right)\right) \\
=\delta^{1} \ldots \delta^{m-l-1} \circ \Delta^{m-l-2} \ldots \Delta^{0} \circ \delta^{1} \ldots \delta^{m-l-1}\left(u_{m-l-1}\right) \\
=\delta^{1} \ldots \delta^{m-l-1} \circ U^{m-l-1}\left(u_{m-l-1}\right)=c_{m-l-1} v_{m-l-1} .
\end{gathered}
$$

So we put

$$
\pi_{a, b}^{m-l-1}:=\frac{1}{c_{m-l-1}}\left(L^{m-l-1}\left(\operatorname{id}_{V^{0}}-\sum_{i=0}^{l} \pi_{a, b}^{m-i}\right)\right) .
$$

Formulas (52), (55), (56), (57), (58), (59) contain the algorithm to compute the $G$-equivariant linear projection

$$
\pi_{a, b}^{i}: V^{0} \rightarrow V^{0}(a-i, b-i) \subset V^{0}
$$


and thus to compute the associated $G$-equivariant bilinear map

$$
\beta_{a, b}^{i}: V(a, 0) \times V(0, b) \rightarrow V(a-i, b-i)
$$

in suitable bases in source and target (remark that $V(a, 0)=\operatorname{Sym}^{a} \mathbb{C}^{3}$ and $\left.V(0, b)=\operatorname{Sym}^{b}\left(\mathbb{C}^{3}\right)^{\vee}\right)$.

In particular, we obtain for $a=2, b=1$ the map

$$
\begin{gathered}
\pi_{2,1}^{0}: V^{0}=\operatorname{Sym}^{2} \mathbb{C}^{3} \otimes\left(\mathbb{C}^{3}\right)^{\vee} \rightarrow V(2,1) \subset V^{0} \\
\pi_{2,1}^{0}=\mathrm{id}-\frac{1}{4} \delta^{1} \Delta^{0}
\end{gathered}
$$

for $a=b=2$ the map

$$
\begin{gathered}
\pi_{2,2}^{0}: V^{0}=\operatorname{Sym}^{2} \mathbb{C}^{3} \otimes \operatorname{Sym}^{2}\left(\mathbb{C}^{3}\right)^{\vee} \rightarrow V(2,2) \subset V^{0} \\
\pi_{2,2}^{0}=\mathrm{id}-\frac{1}{5} \delta^{1} \Delta^{0}+\frac{1}{40} \delta^{1} \delta^{2} \Delta^{1} \Delta^{0}
\end{gathered}
$$

and for $a=b=1$ the map

$$
\begin{gathered}
\pi_{1,1}^{0}: V^{0}=\mathbb{C}^{3} \otimes\left(\mathbb{C}^{3}\right)^{\vee} \rightarrow V(1,1) \subset V^{0} \\
\pi_{1,1}^{0}=\mathrm{id}-\frac{1}{3} \delta^{1} \Delta^{0} .
\end{gathered}
$$

In the following, we will often view elements $x \in V(a, b)$ as tensors $x=\left(x_{j_{1}, \ldots, j_{a}}^{i_{1}, \ldots, i_{b}}\right) \in\left(\mathbb{C}^{3}\right)^{\otimes a} \otimes\left(\mathbb{C}^{3 \vee}\right)^{\otimes b}=: T_{a}^{b} \mathbb{C}^{3}$ (the indices ranging from 1 to 3 ) which are covariant of order $b$ and contravariant of order $a$ via the natural inclusions

$$
V(a, b) \subset \operatorname{Sym}^{a} \mathbb{C}^{3} \otimes \operatorname{Sym}^{b}\left(\mathbb{C}^{3}\right)^{\vee} \subset T_{a}^{b} \mathbb{C}^{3}
$$

(the first inclusion arises since $V(a, b)$ is the kernel of $\Delta^{0}$, the second is a tensor product of symmetrization maps). In particular, we have the determinant tensor det $\in T_{0}^{3} \mathbb{C}^{3}$ and its inverse $\operatorname{det}^{-1} \in T_{3}^{0} \mathbb{C}^{3}$. In formulas involving several tensors, we will also adopt the summation convention throughout. Finally, we define

$$
\begin{gathered}
\operatorname{can}: T_{a}^{b} \mathbb{C}^{3} \rightarrow \operatorname{Sym}^{a} \mathbb{C}^{3} \otimes \operatorname{Sym}^{b}\left(\mathbb{C}^{3}\right)^{\vee} \\
e_{j_{1}} \otimes \cdots \otimes e_{j_{a}} \otimes x_{i_{1}} \otimes \cdots \otimes x_{i_{b}} \mapsto e_{j_{1}} \cdots e_{j_{a}} \otimes x_{i_{1}} \cdots x_{i_{b}}
\end{gathered}
$$

as the canonical projection.

We write down the explicit formulas for the equivariant maps in section 2. The map $\Psi: V(0,4) \rightarrow V(2,2)$ (degree 3 ) is given by

$$
\begin{gathered}
\Psi(f):=\pi_{2,2}^{0}(\operatorname{can}(g)), \\
g_{j_{1} j_{2}}^{i_{1} i_{2}}:=f^{i_{1} i_{2} i_{3} i_{4}} f^{i_{5} i_{6} i_{7} i_{8}} f^{i_{9} i_{10} i_{11} i_{12}} \operatorname{det}_{i_{3} i_{5} i_{9}}^{-1} \operatorname{det}_{i_{4} i_{6} i_{10}}^{-1} \operatorname{det}_{j_{1} i_{7} i_{11}}^{-1} \operatorname{det}_{j_{2} i_{8} i_{12}}^{-1} .
\end{gathered}
$$


The map $\Phi: V(2,2) \times V(0,2) \rightarrow V(2,1)$ (bilinear) is given by

$$
\begin{gathered}
\Phi(g, h):=\pi_{2,1}^{0}(\operatorname{can}(r)), \\
r_{j_{1} j_{2}}^{i_{1}}:=g_{j_{1} i_{3}}^{i_{1} i_{2}} h^{i_{3} i_{4}} \operatorname{det}_{i_{2} i_{4} j_{2}}^{-1} .
\end{gathered}
$$

The map $\epsilon: V(0,4) \times V(0,2) \rightarrow V(2,2)$ (bilinear) is

$$
\epsilon(f, h):=\operatorname{can}(g), g_{j_{1} j_{2}}^{i_{1} i_{2}}:=f^{i_{3} i_{4} i_{1} i_{2}} h^{i_{5} i_{6}} \operatorname{det}_{i_{3} j_{1} i_{5}}^{-1} \operatorname{det}_{i_{4} j_{2} i_{6}}^{-1} .
$$

The map $\zeta: V(0,4) \times V(0,2) \rightarrow V(1,1)$ (homogeneous of degree 2 in both factors) is given by

$$
\zeta(f, h):=\pi_{1,1}^{0}(a),
$$

$$
a_{j_{1}}^{i_{1}}:=h^{i_{1} i_{2}} h^{i_{3} i_{4}} f^{i_{5} i_{6} i_{7} i_{8}} f^{i_{9} i_{10} i_{11} i_{12}} \operatorname{det}_{i_{5} i_{9} j_{1}}^{-1} \operatorname{det}_{i_{6} i_{10} i_{2}}^{-1} \operatorname{det}_{i_{7} i_{11} i_{3}}^{-1} \operatorname{det}_{i_{8} i_{12} i_{4}}^{-1} .
$$

The map $\tilde{\gamma}: V(2,2) \rightarrow V(1,1)$ (homogeneous of degree 2 ) is given by

$$
\tilde{\gamma}:=\pi_{1,1}^{0}(u), u_{j_{1}}^{i_{1}}:=g_{i_{3} i_{4}}^{i_{1} i_{2}} g_{j_{1} i_{2}}^{i_{3} i_{4}} .
$$

\section{Appendix B. Collection of FORMulas for SeCtion 3}

In section 3.1, we saw (formula (26)) that

$$
\delta_{\lambda}=Q_{1}(x, s) a_{1}+Q_{2}(x, s) a_{2}+Q_{3}(x, s) a_{3}+Q_{4}(x, s) a_{4}+Q_{5}(x, s) a_{5} .
$$

We collect here the explicit values of the $Q_{i}(x, s)$ (recall $\lambda=(1,6 \epsilon, 1,6)$, $\epsilon \neq 0)$ :

$$
\begin{aligned}
& Q_{1}(x, s)=\hat{Q}_{1}(x)+2 x_{7} s_{1}+12 x_{8} s_{2}+2 x_{9} s_{1}+\epsilon\left(12 s_{1} s_{2}\right)+2 s_{0} s_{1} \\
& +48 x_{2} s_{4}-48 x_{3} s_{5}-2 x_{4} s_{3}+16 x_{5} s_{4}-16 x_{6} s_{5}+\epsilon\left(-12 s_{4}^{2}-12 s_{5}^{2}\right) \text {, } \\
& Q_{2}(x, s)=\hat{Q}_{2}(x)+4 x_{8} s_{1}+12 x_{9} s_{2}+\epsilon\left(2 s_{1}^{2}-6 s_{2}^{2}\right)+2 s_{0} s_{2} \\
& -4 x_{1} s_{3}+16 x_{2} s_{4}+16 x_{3} s_{5}-16 x_{5} s_{4}-16 x_{6} s_{5}+\epsilon\left(-2 s_{3}^{2}-4 s_{4}^{2}+4 s_{5}^{2}\right) \text {, } \\
& Q_{3}(x, s)=\hat{Q}_{3}(x)+2 x_{4} s_{1}+12 x_{1} s_{2}+64 x_{2} s_{5}+64 x_{3} s_{4} \\
& -2 x_{7} s_{3}+2 x_{9} s_{3}+\epsilon\left(12 s_{2} s_{3}-24 s_{4} s_{5}\right)+2 s_{0} s_{3}, \\
& -8 x_{1} s_{5}-16 x_{3} s_{3}+8 x_{8} s_{4}-8 x_{9} s_{4}+\epsilon\left(-6 s_{1} s_{4}-6 s_{2} s_{4}+6 s_{3} s_{5}\right)+2 s_{0} s_{4} \text {, } \\
& Q_{5}(x, s)=\hat{Q}_{5}(x)+4 x_{6} s_{1}+12 x_{3} s_{1}+12 x_{6} s_{2}-12 x_{3} s_{2} \\
& +8 x_{1} s_{4}-16 x_{2} s_{3}-8 x_{8} s_{5}-8 x_{9} s_{5}+\epsilon\left(6 s_{1} s_{5}-6 s_{2} s_{5}-6 s_{3} s_{4}\right)+2 s_{0} s_{5} \text {, }
\end{aligned}
$$


where

$$
\begin{gathered}
\hat{Q}_{1}(x)=-192 x_{6}^{2}-192 x_{3} x_{6}+384 x_{3}^{2}-192 x_{5}^{2}-192 x_{2} x_{5}+384 x_{2}^{2} \\
-12 x_{1} x_{4}+12 x_{7} x_{8}+180 x_{8} x_{9} \\
\hat{Q}_{2}(x)=64 x_{6}^{2}-192 x_{3} x_{6}-128 x_{3}^{2}-64 x_{5}^{2}+192 x_{2} x_{5}+128 x_{2}^{2} \\
-2 x_{4}^{2}+16 x_{1}^{2}+2 x_{7}^{2}-16 x_{8}^{2}-50 x_{9}^{2} \\
\hat{Q}_{3}(x)=96 x_{5} x_{6}-672 x_{3} x_{5}-672 x_{2} x_{6}+1248 x_{2} x_{3} \\
-12 x_{1} x_{7}+12 x_{4} x_{8}+180 x_{1} x_{9} \\
\hat{Q}_{4}(x)=6 x_{4} x_{6}+42 x_{3} x_{4}+84 x_{1} x_{6}+156 x_{1} x_{3} \\
-6 x_{5} x_{7}-42 x_{2} x_{7}+24 x_{5} x_{8}-264 x_{2} x_{8}+30 x_{5} x_{9}-30 x_{2} x_{9} \\
\hat{Q}_{5}(x)=-6 x_{4} x_{5}-42 x_{2} x_{4}+84 x_{1} x_{5}+156 x_{1} x_{2} \\
+6 x_{6} x_{7}+42 x_{3} x_{7}+24 x_{6} x_{8}-264 x_{3} x_{8}-20 x_{6} x_{9}+30 x_{3} x_{9} .
\end{gathered}
$$

The polynomials $q_{1}, \ldots, q_{5}$ defining $\tilde{Y}_{\lambda} \subset R \times \mathbb{P}^{8}$ (cf. Theorem 3.2.1) are:

$$
\begin{gathered}
q_{1}=\left(-192 r_{3}^{2}-192 r_{3}+384\right) y_{1} y_{2}+\left(-192 r_{2}^{2}-192 r_{2}+384\right) y_{1} y_{3} \\
+\left(-12 r_{1}\right) y_{2} y_{3}+12 y_{7} y_{8}+180 y_{8} y_{9}+2 y_{7} y_{11}+12 y_{8} y_{12} \\
+2 y_{9} y_{1} 1+\epsilon\left(12 y_{11} y_{12}\right)+2 y_{10} y_{11} \\
q_{2}=\left(64 r_{3}^{2}-192 r_{3}-128\right) y_{1} y_{2}+\left(-64 r_{2}^{2}+192 r_{2}+128\right) y_{1} y_{3} \\
+\left(-2 r_{1}^{2}+16\right) y_{2} y_{3}+2 y_{7}^{2}-16 y_{8}^{2}-50 y_{9}^{2}+4 y_{8} y_{11}+12 y_{9} y_{12} \\
+\epsilon\left(2 y_{11}^{2}-6 y_{12}^{2}\right)+2 y_{10} y_{12} \\
q_{3}=\left(96 r_{2} r_{3}-672 r_{2}-672 r_{3}+1248\right) y_{1} \\
-12 y_{7}+12 r_{1} y_{8}+180 y_{9}+2 r_{1} y_{11}+12 y_{12}
\end{gathered}
$$

$$
\begin{gathered}
q_{4}=\left(6 r_{1} r_{3}+42 r_{1}+84 r_{3}+156\right) y_{2}+\left(-6 r_{2}-42\right) y_{7}+\left(24 r_{2}-264\right) y_{8} \\
+\left(30 r_{2}-30\right) y_{9}+\left(4 r_{2}+12\right) y_{11}+\left(-12 r_{2}+12\right) y_{12}, \\
\begin{array}{c}
(84) \\
q_{5}=\left(-6 r_{1} r_{2}-42 r_{1}+84 r_{2}+156\right) y_{3}+\left(6 r_{1}+42\right) y_{7}+\left(24 r_{3}-264\right) y_{8} \\
+\left(-30 r_{3}+30\right) y_{9}+\left(4 r_{3}+12\right) y_{11}+\left(12 r_{3}-12\right) y_{12} .
\end{array}
\end{gathered}
$$

\section{REFERENCES}

[Bog1] Bogomolov, F.A., Rationality of the moduli of hyperelliptic curves of arbitrary genus, in: Proceedings of the 1984 Vancouver Conference in Algebraic 
Geometry, CMS Conference Proceedings, vol. 6, American Math. Society (1986), 17-37

[B-S] Brion, M. \& Schwarz, G.W., Théorie des invariants 83 Géométrie des variétés quotients, Collection Travaux en Cours, Hermann Éditeurs des Sciences et des Arts, Paris (2000)

[Cl] Clebsch, A., Theorie der binären algebraischen Formen, B. G. Teubner, Leipzig (1872)

[C-L] Clebsch, A. \& Lindemann, F., Vorlesungen über Geometrie, Erster Band. Geometrie der Ebene. B. G. Teubner, Leipzig (1876)

[Dix] Dixmier, J., On the Projective Invariants of Quartic Plane Curves, Adv. in Math. 64 (1987), 279-304

[Dol1] Dolgachev, I., Rationality of fields of invariants, in Algebraic Geometry, Bowdoin, Proc. Symp. Pure Math. vol. 46 (1987), 3-16

[Dol2] Dolgachev, I., Lectures on Invariant Theory, London Mathematical Society Lecture Note Series 296, Cambridge Univ. Press (2003)

[Dol3] Dolgachev, I., Topics in Classical Algebraic Geometry. Part I, available at http://www.math.lsa.umich.edu/ idolga/lecturenotes.html

[D-O] Dolgachev, I., Ortland, D., Point Sets in Projective Space and Theta Functions, Astérisque, vol. 165 (1989)

[Fu-Ha] Fulton, W. \& Harris, J., Representation Theory. A First Course, Springer G.T.M. 129, Springer-Verlag (1991)

[G-Y] Grace, J.H. \& Young, W.H., The Algebra of Invariants, Cambridge Univ. Press (1903); reprinted by Chelsea Publ. Co. New York (1965)

[Kat0] Katsylo, P. I., Rationality of the moduli variety of curves of genus 5, Math. USSR Sbornik, vol. 72 (1992), no. 2, 439-445

[Kat1] Katsylo, P.I., On the birational geometry of the space of ternary quartics, Advances in Soviet Math. 8 (1992), 95-103

[Kat2] Katsylo, P.I., Rationality of the moduli variety of curves of genus 3, Comment. Math. Helvetici 71 (1996), 507-524

[Muk] Mukai, S., An Introduction to Invariants and Moduli, Cambridge studies in advanced mathematics 81, Cambridge Univ. Press (2003)

[Mum] Mumford, D., Fogarty, J., Kirwan, F., Geometric Invariant Theory, Third Enlarged Edition, Ergebnisse der Mathematik und ihrer Grenzgebiete 34, Springer-Verlag (1994)

[Ol] Olver, P. J., Classical Invariant Theory, London Mathematical Society Student Texts 44, Cambridge University Press (1999)

[Po-Vi] Popov, V.L. \& Vinberg, E.B., Invariant Theory, in: Algebraic Geometry IV, A.N. Parshin, I.R. Shafarevich (eds.), Encyclopedia of Mathematical Sciences, vol. 55, Springer Verlag (1994)

[Pro] Procesi, C., Lie Groups. An Approach through Invariants and Representations, Springer Universitext, Springer-Verlag (2007)

[Sal] Salmon, G., A Treatise on the Higher Plane Curves, Hodges, Foster and Figgis, (1879); reprinted by Chelsea Publ. Co. (1960)

[Se] Serre, J.-P., Linear Representations of Finite Groups, Springer GTM 42, Springer-Verlag (1977)

[Wey] Weyman, J., The Equations of Strata for Binary Forms, Journal of Algebra 122 (1989), 244-249 